\title{
On Air Entrainment in a Water pool by Impingement of a
}

\author{
Jet \\ Vatsal Sanjay and Arup Kumar Das* \\ Department of Mechanical and Industrial Engineering \\ Indian Institute of Technology, Roorkee - 247667 \\ *arupdas80@gmail.com
}

This is the pre-print version of the following article: [Sanjay V, Das AK. On air entrainment in a water pool by impingement of a jet. AIChE Journal;63(11):51695181], which has been published in final form at DOI: 10.1002/aic.15828. This article may be used for non-commercial purposes in accordance with Wiley Terms and Conditions for Self-Archiving

\begin{abstract}
Air entrainment due to impingement of a water jet on a pool is studied extensively to understand the physics of the initiation and the cluster of bubbles formed below the free surface. Possible outcomes due to the jet impingement in a pool have been identified as smooth free surface without entrainment or formation of rigorous bubble cluster below the jet-pool contact. Triangular entrained region is found to be a three-dimensional association of disconnected bubble population continuously breaking and making with the neighbors. A correlation for prediction of maximum entrained height for a range of jet diameters and lengths is proposed. The trajectory of a single bubble is also studied to understand the kinematics of the bubble cluster. Alongside, an electrical conductivity probe has been used to examine the probabilistic presence of the bubble at a given depth in the liquid pool.
\end{abstract}

Keywords: Entrainment, Jet - Pool Interaction, Interface dynamics 


\section{Introduction}

Entrainment of air inside a liquid pool by impinging jet is not just one of the widely encountered phenomenon in nature but also has several industrial applications. A look around reveals the presence of this phenomenon in everyday life, ranging from filling of a glass of water using tap to several chemical reactions, such as mineral flotation, steel teeming process and gas absorption. Evan et al. ${ }^{2}$ defines a plunging liquid jet as a moving column of liquid that passes through a gaseous head-space before impinging on the horizontal free surface. In the wake of its common occurrence, this phenomenon has attracted researchers' attention all across the past century. In his review work, Biń ${ }^{3}$ reviewed previous works in the area of impinging jet in a pool and showed application of the same in the field of wastewater treatment. Entrainment is desirable in processes like aeration reactions during waste water treatment whereas it is undesirable in processes such as pouring of molten metals during casting or glass molding. Hence, it is important to understand the physics behind this phenomenon so that we can optimize the processes it affects, directly or indirectly. There have been several attempts at understanding the flow patterns caused by two-dimensional plunging liquid jets $\frac{45}{4}$. However, from an industrial point of view, three dimensional jets are more important since they are easy to achieve and hence has been widely studied by researchers. Nevertheless, there have been only a few studies on the flow fields just below the impingements. Most of the works revolve around what Bonetto and Lahey ${ }^{6}$ termed as the global measurements. Among those are the works of Mckeogh ${ }^{17}$, Donk ${ }^{8}$ and El Hammoumi et al. ${ }^{9}$, who recorded the air concentration profiles and velocity distribution in the fully developed flows. As identified by Evans et al. ${ }^{2}$, gas entrainment rate depends on the effective diameter of the free jet at the plunge point and the annular film thickness adjacent to the surface of the jet. Undoubtedly this study is important to have a control over the process and these studies may allow correlation of the onset of air-carry under criteria, very little or no information is provided about the kinematics and dynamics of the entrained bubbles and the resultant dispersion of the submerged jet. Even though this process is so common in nature, the mechanism involved is not well understood. Entrainment characteristics need to be related with flow rate, jet diameter and length of jet for better understanding.

Along with the review works of Bin ${ }^{3}$ and Chanson $\frac{10}{10}$, the report by Oguz et al. ${ }^{11}$ containing sev- 
eral examples of air-entraining flows, pioneered the research in this field. As the jet impacts the free liquid surface, there is a chance that the turbulent or laminar jet entrain bubbles, provided the velocity of impact is more than the threshold velocity for entrainment as pointed by Lin and Donnelly ${ }^{12}$. This onset value depends on the disturbances present on the jet surface itself ${ }^{4}$. Bin $^{13}$ and Fetisov ${ }^{14}$ have developed empirical relations for determination of critical impact velocities for the onset of entrainment phenomenon. Near inception flow conditions were revealed by Cummings and Chanson ${ }^{15}$. In another work, they ${ }^{[16}$ also discussed some of the characteristics of air bubbles entrained by smooth jets. Further, they studied extensively the effects of velocity on the air entrainment phenomenon and its quantity along with coalescence and breaking of individual bubbles ${ }^{17}$. An analytical approach to the problem can be found in the work of Soh

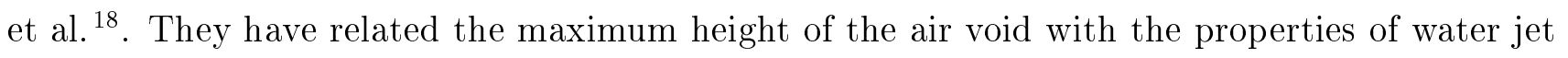
using the energy balance method. However, the method is too idealized, neglecting the losses and simplification of geometry. The theory seems to be in good agreement with the experimental results only for low diametrical Froude numbers $\left(F r_{D}<10\right.$, where, $\left.F r_{D}=\frac{V_{j}}{\sqrt{g d_{j}}}\right)$ with an error as high as $50 \%$ for $\mathrm{Fr}_{D}>50$. Chanson et al. $\frac{19}{19}$ investigated air entrainment and bubble dispersion in the developing vertical circular plunging jets and came up with some remarkable results and expressed a requirement to study the air-water velocity distribution and turbulent velocity fluctuations. Clanet and Lasheras ${ }^{20}$ reported some experiments conducted with round plunging jets that reveal interesting results concerning the depth of penetration of the bubble cloud under a wide range of jet diameters, velocities and plunging angles. Irrespective of such attempts at development of correlations, as argued by Van de Sande and Smith ${ }^{21}$, a lack of generality is observed (discrepancies of the order of 3 or more). It has been observed that the parameters proposed by one investigator is off by noticeable amount when compared to data from others. This coupled with problems in directly solving the governing equations analytically justifies our approach to the problem numerically.

Efforts are also being noticed to understand the physics of entrainment using numerical approach. Qu et al. ${ }^{22}$ published a combined experimental and numerical investigation. They studied the mixture approach against the level-set approach for flow modeling. Since, this phenomenon rigorously involves the consideration of interface and its deformations with time, level-set approach has an edge over the mixture approach. Since, Volume of Fluid (VOF) specializes in implicit 
interface capturing characteristics, it will be even more suited for the solution of entrainment problems. As mentioned by Chen and Hagen ${ }^{23}$, VOF captures the topology change of the moving surfaces, such as merging and breakup of bubbles. The research paper by Deshpande and Trujillo ${ }^{24}$ comprises of the distinguishing features of the shallow angle plunging jets using VOF methodology. However, the work deals with inclined jets and till the pinch off moment for vertical jets. Brouilliot and Lubin ${ }^{25}$ reported remarkable compliance of numerical and experimental results with classical VOF-PLIC method. At the same time limitations of this method to account for inclusions smaller that the mesh grid size can be observed. Durve and Patwardhan 26 outlined the onset of gas entrainment through numerical simulations. It can be easily established that over the past couple of decades, the main focus has been to explore the mechanism of the entrainment process. Zhu et al. ${ }^{[27}$ studied the mechanism of air entrainment due to surface roughness. However, the work involves numerical simulations only up to the impact and pinch off point. Recent study complies with the proposed air cavity formation reported in the above paper.

Kersten et al. ${ }^{28}$ examined the spread of falling liquid over the receiving pool, formation of cavity and eventual pinch off. The initial bubble that pinches off after the closure of cavity and the air sheathe around the submerged jet can be designated as the source of bubbles inside the pool. Recently, comprehensive research has been carried out on further characterization of different parameters related to entrainment $29|30| 31$. Several researchers have concluded the requirement for the study of influence of the controlling parameters, such as jet length and the velocity on penetration depth and entrainment volume ${ }^{32}$. Roy et al. $\stackrel{33}{3}$ examined descriptively the trajectory of a single bubble in the entrainment region. However, the literature still lacks an exhaustive account for the analysis of bubble life cycle during entrainment.

Moreover, there have been very little work on the characterization of the region affected in the liquid. In our investigation, we have focused on the characterization of the cluster of bubbles and the region of entrainment in the liquid pool. Dynamic behavior of the interface cluster is studied and observations have been carried out to record the variation in shape with time starting from the point of inception of entrainment. Attempts are also made to understand the formation of air cavity and subsequent pinch off details along with fragmentation of the initial bubble into smaller bubbles. Discussions are carried out for the kinematics and dynamics of 


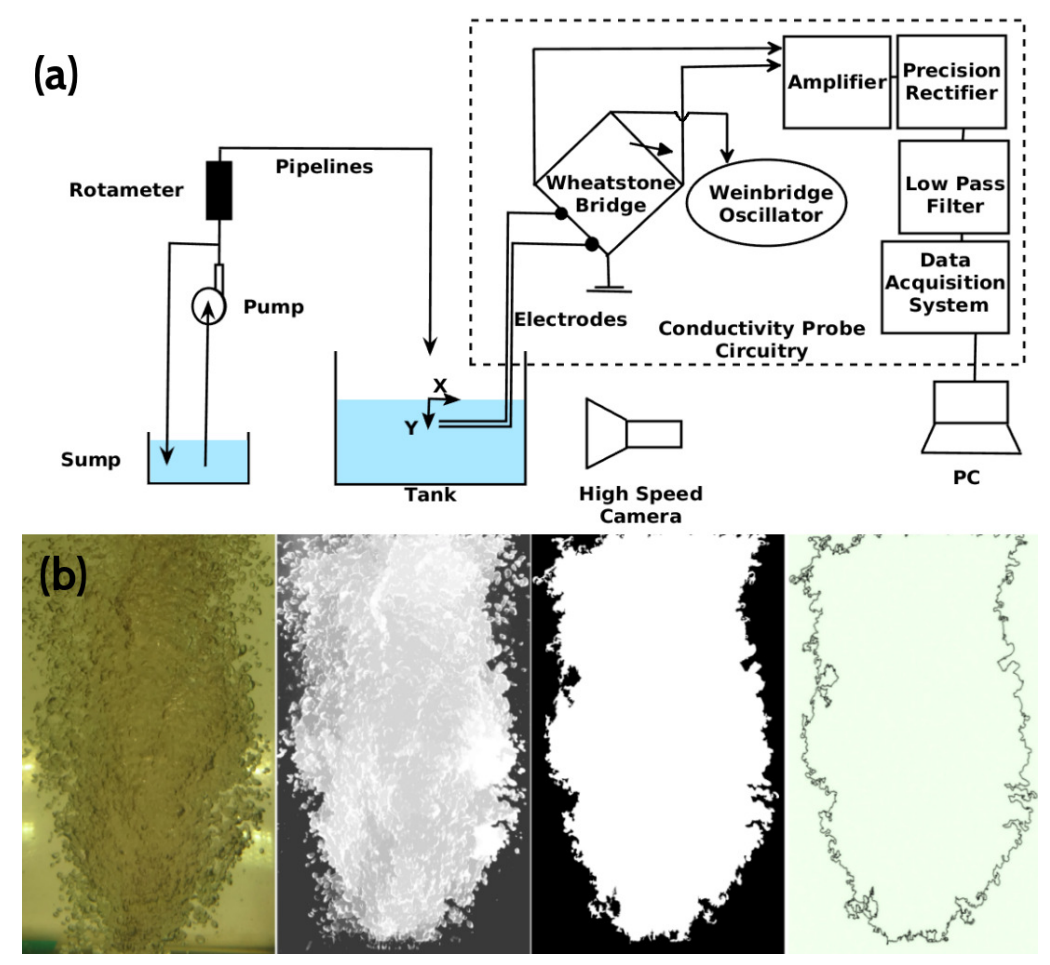

Figure 1: (a) In house developed experimental setup for the study of entrainment process. The coordinate system has been defined using the Cartesian system centered at the point of impact of the jet on the pool. (b) Sequence of image processing applied for the characterization of the bubble cluster.

bubble, commencing at the time of entrainment to its trajectory inside and outside the bubble cluster to the instance when it erupts at the water surface. A conductivity probe has been used in order to obtain strength of entrainment at different flow rate. Both visualization and signal conditioning established regions of higher entrainment areas to shed light for control of complex inter-facial interactions. The details of these experimental methods and our setup are presented in the next section.

\section{Experimental Setup and Methodology}

Figure 1(a) illustrates the schematic of the experimental setup used for the present study. A water pool in a Plexiglas tank $(0.5 \mathrm{~m} \times 0.5 \mathrm{~m} \times 0.5 \mathrm{~m})$, is impinged by a water jet (with properties as given in Table 1). Flow of the jet is controlled using valves and is measured with a calibrated rota-meter at ambient conditions. Arrangements are made to lower the jet for variation of its height from the pool $\left(l_{j}\right)$. Further, the jet diameter $\left(d_{j}\right)$ can be changed by using different guiding nozzles. Injection system is kept as sufficient long $(\sim 120 D)$ to obtain fully developed 


\begin{tabular}{cccc}
\hline Fluid & $\begin{array}{c}\text { Density } \\
\left(\mathrm{kg} / \mathrm{m}^{3}\right)\end{array}$ & $\begin{array}{c}\text { Viscosity } \\
(\text { Pas })\end{array}$ & $\begin{array}{c}\text { Surface Tension } \\
(\mathrm{Nm})\end{array}$ \\
\hline Water & 1000 & 0.001 & 0.072 \\
Air & 1.24 & $1.8 \mathrm{e}-5$ & \\
\hline
\end{tabular}

Table 1: Details of the physical properties of the tap water used in the experiments and the ambient air conditions.

\begin{tabular}{cccc}
\hline Flow & Nozzle & Reynold's & Froude \\
Rate & Diameter & Number & Numbers \\
$\left(\mathrm{x} 10^{-5} \mathrm{~m}^{3} / \mathrm{s}\right)$ & $(\mathrm{m})$ & $R e$ & $\mathrm{Fr}_{D} F r_{L}$ \\
\hline 1.67 to 100 & 0.01 to 0.0256 & 2000 to 50000 & 1 to 4 \\
\hline
\end{tabular}

Table 2: Variation of different parameters associated with the flow. The Reynolds number and the product of Froude numbers are based on jet characteristics

velocity profile in the injected water jet.

Noticing the dynamics of the problem, it is realized that the depth of the entrainment $\left(d_{s}\right)$ is dependent on the kinematic or geometric control parameters, such as the diameter of the jet $\left(d_{j}\right)$, velocity of the jet $\left(V_{j}\right)$, length of the jet $\left(l_{j}\right)$ along with other physical variables such as the density of the liquid $\left(\rho_{l}\right)$, its viscosity $\left(\mu_{l}\right)$, surface tension, $\sigma$ and acceleration due to gravity $(g)$.

$$
d_{s}=\Phi\left(V_{j}, d_{j}, \rho_{l}, \mu, \sigma, \vec{g}\right)
$$

Assuming, $V_{j}, d_{j}$ and $\rho_{l}$ as the repeating variables in Equation 1, we reach at the functional relation shown in Equation 2 .

$$
\frac{d_{s}}{d_{j}}=\Psi\left(\frac{\sqrt{g d_{j}}}{V_{j}}, \frac{l_{j}}{d_{j}}, \frac{\mu}{\rho_{l} V_{j} d_{j}}, \frac{\sigma}{\rho_{l} V_{j}^{2} d_{j}}\right)
$$

The first term (inverse of the diametrical Froude number, $F r_{D}$ ) is a measure of the inertia of the circular jet whereas the second term is directly associated with the gravitational potential available to the jet as it starts its descend towards the pool. Further, the last two terms can be identified as the inverse of the Reynolds and the Weber number. The diametrical Froude Number $\left(F r_{D}\right)$ and the jet length ratio $\left(\frac{l_{j}}{d_{j}}\right)$ present the control aspects of our experiments and simulation. These terms can be combined into the product of the Froude Numbers and the uniqueness of a particular flow configuration is considered using the number, $\operatorname{Fr}_{D} F r_{L}=\left[\frac{V}{\sqrt{g D_{j}}}\right]\left[\frac{V}{\sqrt{g L_{j}}}\right]$, where $F r_{L}$ is the longitudinal Froude Number. Table 2 contains the range of variations involved in the 


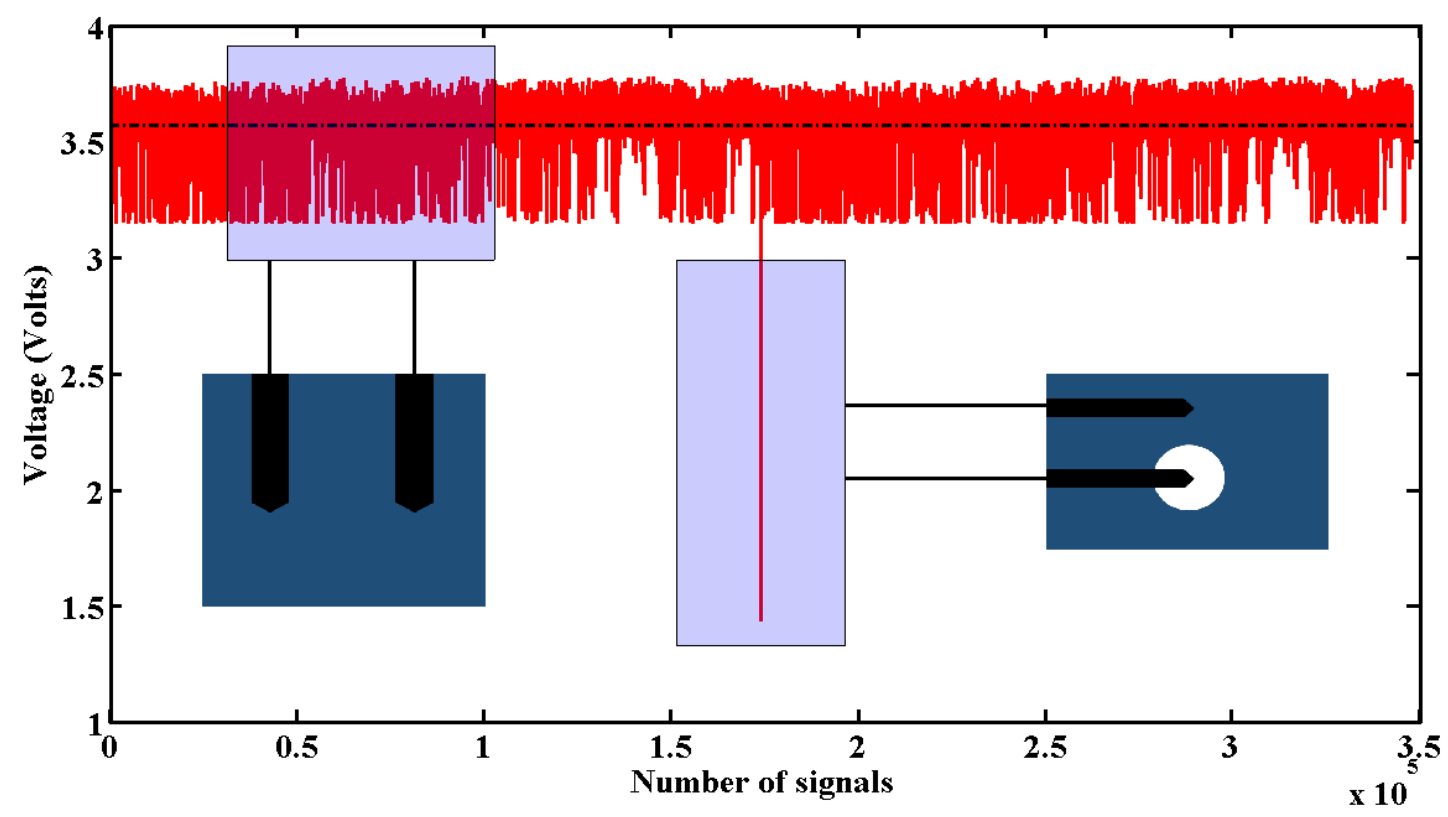

Figure 2: Signal behavior for different closure of circuitry. In case water closes the circuit the voltage signal is normal. However, with the presence of bubbles, an instantaneous dip can be observed

present study. High speed camera is used to capture the dynamic behavior of the entrainment process with a maximum frame rate of 2000 frames per second at full resolution. The raw images obtained from high speed camera are passed through RGB to grayscale conversion, noise removal by subtracting background and grayscale to black and white conversion stages. From temporal, black and white images feature extraction like centre of mass evaluation and area determination of cluster are performed. Sobel technique is used for interface tracking using which is maximum depth of entrainment penetration is determined. Figure 1(b) illustrates different stages of image analysis.

Moreover, an electrical conductivity probe is developed to detect the presence of entrapped air bubbles at a given spatial coordinate in the liquid pool. The working principle of conductivity probe is variable resistance when different (air/water) medium closes the circuitry between two copper electrodes as illustrated in Figure 1(a). To convert the instantaneous variable resistance into measurable voltage signal, electronic circuitry has also been developed. Figure 2 gives an idea about the behavior of probe signals for different media completing the circuitry in between the electrodes. In the next section, we have explained the Mathematical formulation used for spatio-temporal fully resolved simulation of the process. Care has been taken to accommodate at least 10 cells inside disconnected interface for accurate capture of the interfacial dynamics. 
No turbulence model is used in the model framework.

\section{Mathematical Formulation and Domain Description}

Bubble entrainment is studied in a three dimensional finite volume framework using the Volume Of Fluid (VOF) approach for interface tracking. Open source solver, Gerris is used for the study $\stackrel{34}{ }$. It implements the finite volume discretization on an octree adaptive grid with piecewise linear VOF model. $\alpha\left(x_{i}, t\right)$ is taken as the volume fraction (tracer) of water phase at spatial and temporal instance of $x_{i}$ and $t$ respectively. The density and viscosity field for the study can be defined as:

$$
A(\bar{\alpha})=\bar{\alpha} A_{1}+(1-\bar{\alpha}) A_{2} \quad \forall A \in\{\rho, \mu\}
$$

In the Equation $3, \bar{\alpha}$ is calculated through bilinear interpolation of $\alpha\left(x_{i}, t\right)$ from cell centered values using spatial filtering. Mass conservation across the interface is implemented following Equation 4

$$
\frac{\partial V_{i}}{\partial x_{i}}=0
$$

To tackle individual phases, a Volume of Fluid tracer advection has been solved, as described in Equation 5 .

$$
\frac{\partial \alpha}{\partial t}+\frac{\partial\left(\alpha V_{i}\right)}{\partial x_{i}}=0
$$

The temperature variations are assumed to be too small to affect the investigated phenomenon. As a result, no thermodynamic conservation equation is solved. However, the incompressible Navier-Stokes equations are solved for all three spatial coordinates. Equation 6 has the representation for the same in Cartesian tensor notation.

$$
\rho\left(\frac{\partial V_{i}}{\partial t}+V_{j} \frac{\partial V_{i}}{\partial x_{j}}\right)=-\frac{\partial p}{\partial x_{i}}+\frac{\partial\left(2 \mu D_{i j}\right)}{\partial x_{j}}+\sigma \kappa \delta_{s} m_{i}+\rho g_{i}
$$

$\frac{\partial p}{\partial x_{i}}$ refers to the pressure gradient for the flow and $\rho g_{i}$ is the gravitational force applied at a given coordinate in space and time. Moreover, $\kappa, \sigma$ and $m_{i}$ are the curvature of the interface, surface tension force per unit length and the interface normal vector respectively. The multiplication of Dirac distribution function with the surface tension term is to ensure that this force is 


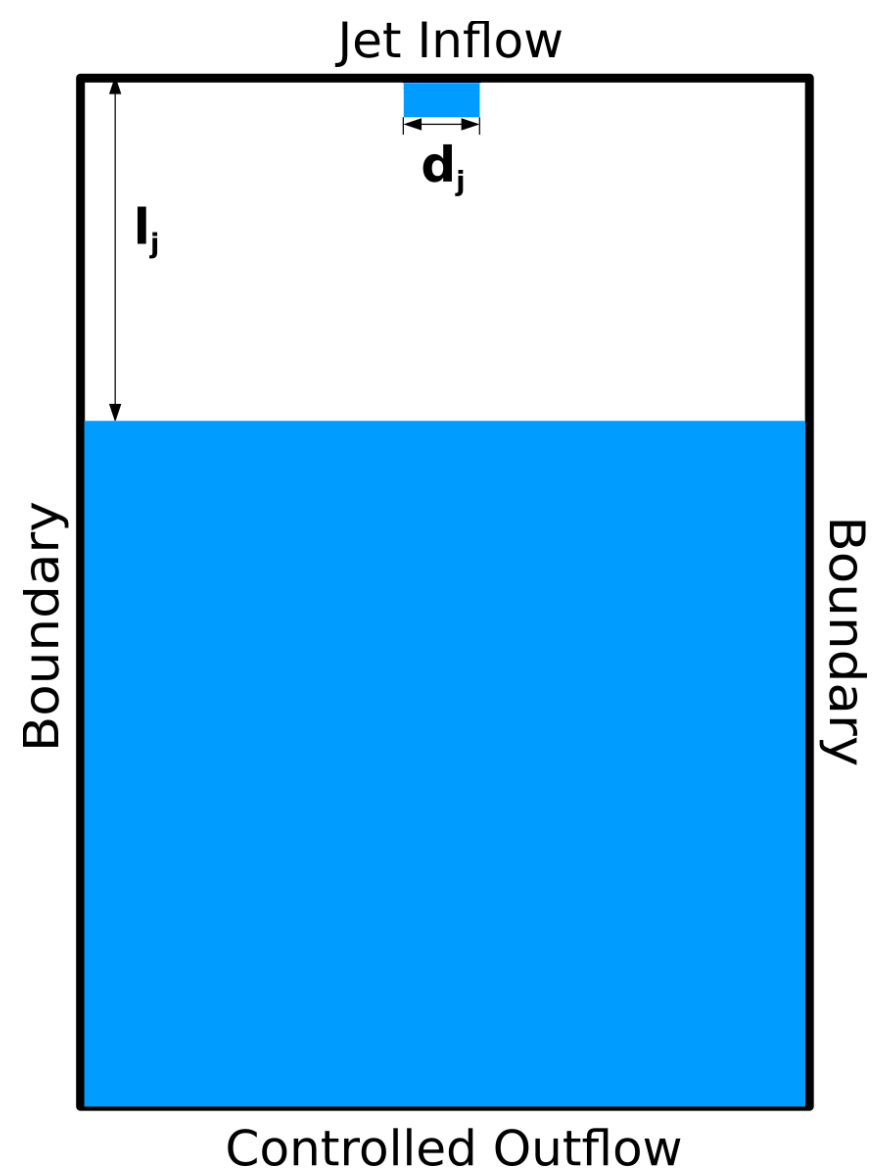

Figure 3: The computational domain with the implemented boundary conditions.

concentrated at the surface of the two phases. Further, the deformation tensor $D_{i j}$ is defined in Equation 7 .

$$
D_{i j}=\frac{1}{2}\left(\frac{\partial V_{i}}{\partial x_{j}}+\frac{\partial V_{j}}{\partial x_{i}}\right)
$$

Second order accurate time discretization of momentum and continuity equations are carried out with time splitting algorithm as proposed by Chorin $\frac{\sqrt{35}}{}$. Gerris decides time step based on Courant-Friedrichs-Lewy (CFL) stability criteria $(\mathrm{CFL}<1)$ employed for Bell-Colella-Glaz second-order unsplit upwind scheme for the estimation of the velocity advection term, $\left(V_{j} \frac{\partial V_{i}}{\partial x_{j}}\right) \frac{36}{\text {. }}$. However, a maximum limit of $5 \times 10^{-4}$ seconds is set to keep the solution in bounds.

Figure 3 illustrates the computational domain with dimensions $30 d_{j} \times 10 d_{j} \times 10 d_{j}$. The lateral surfaces are modeled as boundaries using no slip condition. The distance of these walls from the centerline of the jet $\left(5 d_{j}\right)$ is kept large to avoid any biasing from boundaries. The bottom boundary is given a controlled velocity out-flux condition, to ensure that the water level does not change whereas the top boundary has jet inflow and outflow boundary condition. A small jet is initialized at the start of the simulation, along with the water pool. The product of the Froude 


\begin{tabular}{ccccc}
\hline Case & $\left(\frac{d_{j}}{\delta l}\right)_{\max }$ & $\begin{array}{c}\text { Maximum } \\
\text { level }\end{array}$ & $\left(\frac{d_{j}}{\delta l}\right)_{\min }$ & $\begin{array}{c}\text { Minimum } \\
\text { level }\end{array}$ \\
\hline 1. & 6.4 & 6 & 1.6 & 4 \\
2. & 12.8 & 7 & 1.6 & 4 \\
3. & 25.6 & 8 & 1.6 & 4 \\
4. & 51.2 & 9 & 1.6 & 4 \\
5. & 102.4 & 10 & 1.6 & 4 \\
6. & 204.8 & 11 & 1.6 & 4 \\
7. & 102.4 & 10 & 3.2 & 5 \\
8. & 102.4 & 10 & 6.4 & 6 \\
9. & 102.4 & 10 & 12.8 & 7 \\
10. & 102.4 & 10 & 25.6 & 8 \\
\hline
\end{tabular}

Table 3: Designation of cases considered for the grid sensitivity analysis.

numbers $\left(F r_{D} F r_{L}\right)$ determines the average inlet velocity of the liquid jet and the velocity profile depends on the turbulent characteristic of the jet. If the Reynolds number of the jet is below 2300 (laminar jet), parabolic profile $\left[2\left(1-\left(\frac{2 r}{d_{j}}\right)^{2}\right)\right] V_{j}$ is patched whereas power law velocity profile $\left[\frac{8}{7}\left(1-\frac{2 r}{d_{j}}\right)^{\frac{1}{7}}\right] V_{j}$ is used for turbulent jets. With the above Mathematical model in place, mesh sensitivity analysis and validation of the numerical code is carried out. The results of the same have been presented in the next section.

\section{Grid Independence Study and Validation}

Refinement is bestowed adaptively based on the gradient of the volume fraction of fluid $\alpha\left(x_{i}, t\right)$. This ensures that the number of cells around the interface is sufficient enough to capture small scale variations. Let, $\delta l$ be the size of a cell, which is non-dimensionalized as $\frac{d_{j}}{\delta l}$, a measure of the total number of cells across the diameter of the jet. Keeping the minimum $\frac{d_{j}}{\delta l}$ constant, the maximum $\frac{d_{j}}{\delta l}$ is varied from 204.8 to 6.4 (Level 11 and 6 respectively in Gerris 3D simulation) as cataloged in Table 3. On achievement of consistency in the result for time taken (non dimensionalized as $\frac{V_{j} t}{d_{j}}$ ) to first pinch off, the minimum $\frac{d_{j}}{\delta l}$ is varied. It must be noted that the minimum value of $\frac{d_{j}}{\delta l}$ is never reached near the interface as the interface is always well resolved with maximum refinement available. All the considered cases are given in Table 3 . The parameters for these cases are kept constant at $F r_{D} F r_{L}=1.4$. Figure 4 shows the consequences of this analysis. It can clearly observed that the result saturates after the fourth case. From CPU 


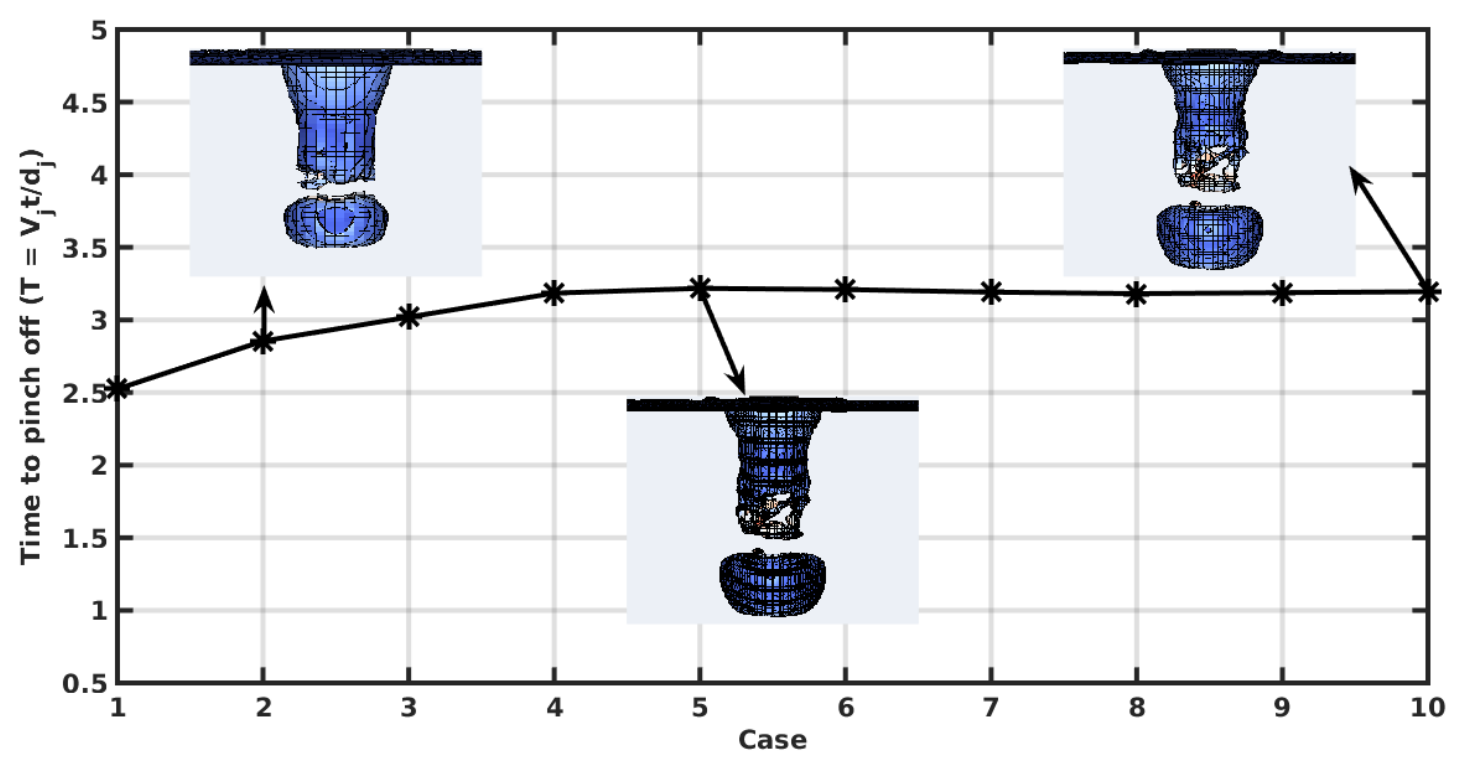

Figure 4: Determination of time taken for pinch off of first bubble after impact on the pool. The in-box figures represent instant of the pinch off. The $F r_{D} F r_{L}=1.4$ and $R e=8000$ are kept constant. Interfacial grids obtained by full Adaptive Mesh Refinement (AMR) are shown in the inset corresponding to case 2 with a relatively coarse grid as well as in case 5 and case 10 with fine grids.

\begin{tabular}{ccc}
\hline Case & $\left(\frac{d_{j}}{\delta l}\right)_{\max }$ & $\begin{array}{l}\left(\frac{t_{C P U}}{t_{\text {actual }}}\right) \\
(\text { days } / \mathrm{s})\end{array}$ \\
\hline 4 & 51.2 & $\sim 7.5$ \\
5 & 102.4 & $\sim 10$ \\
6 & 204.8 & $\sim 15.5$ \\
\hline
\end{tabular}

Table 4: CPU performance data for simulations done for the Grid Independence Study. The simulations are done using four Intel Core i7-6500U CPU having clock speed of $2.5 \mathrm{GHz}$ each and 8 GB RAM.

performance data of Table 4 , one can note that case 5 can be adopted as optimum grid structure keeping compromise between accuracy and runtime.

Figure 5 shows the velocity profile at different locations across the jet, out of which location 1 is very near to the boundary where power law velocity profile has been patched (as described earlier in the mathematical formulation). Downstream to this, point 2 lies outside the liquid pool and has a fully developed water jet with high magnitude of velocities near the periphery where air is present. A part of jet's initial gravitational potential has been converted into its kinetic energy resulting in higher velocity and lower instantaneous diameter. Other points are inside the pool with location 3 in the region surrounded by the thin air sheathe whose small velocity is reflected near the interface of the jet. Though the jet is inside the pool, it still retains 
(a)

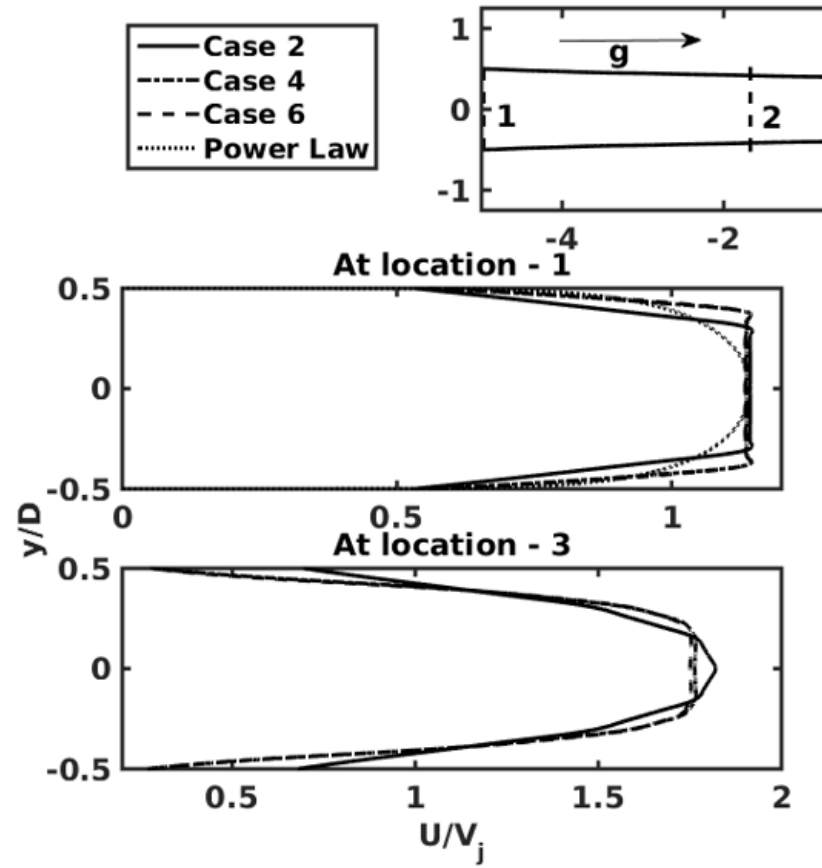

(b)

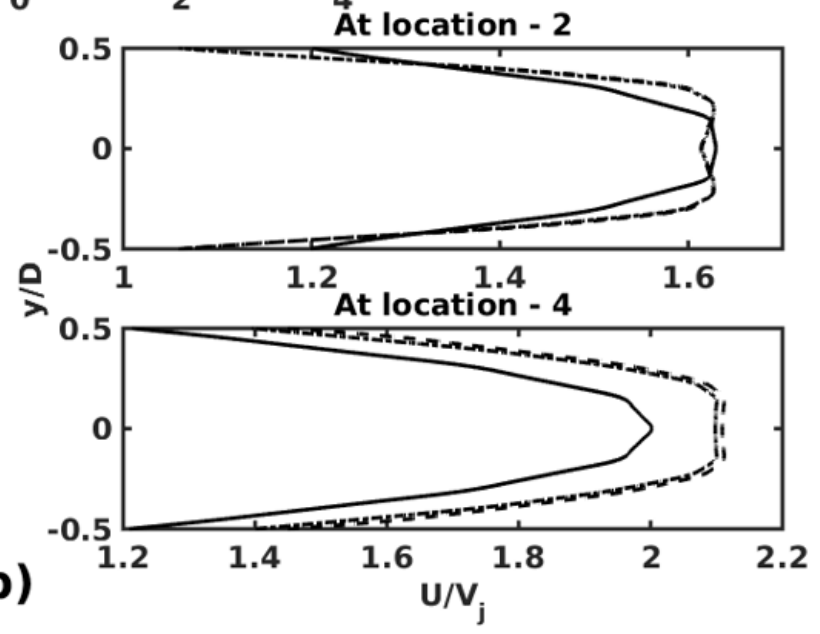

Figure 5: (a) Schematic of the different locations where the velocity profile is plotted and compared. The phase boundary corresponds to that of case 6 . (b) Velocity profile as observed at indicated locations. $\left(F r_{D} F r_{L}=1.4\right.$ and $\left.T=\frac{V_{j} t}{d_{j}}=2.5\right)$

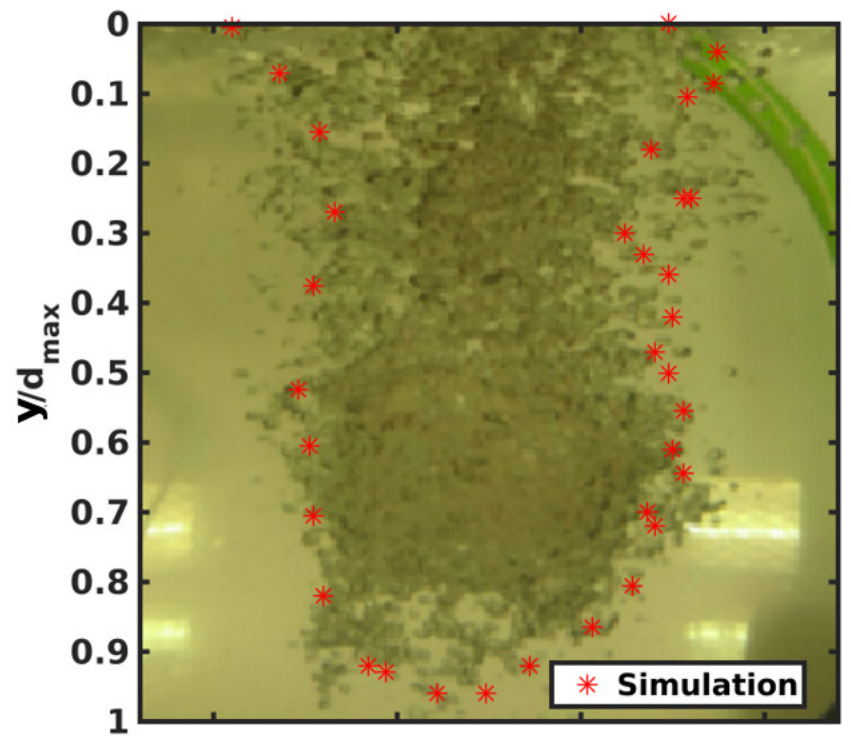

(a)

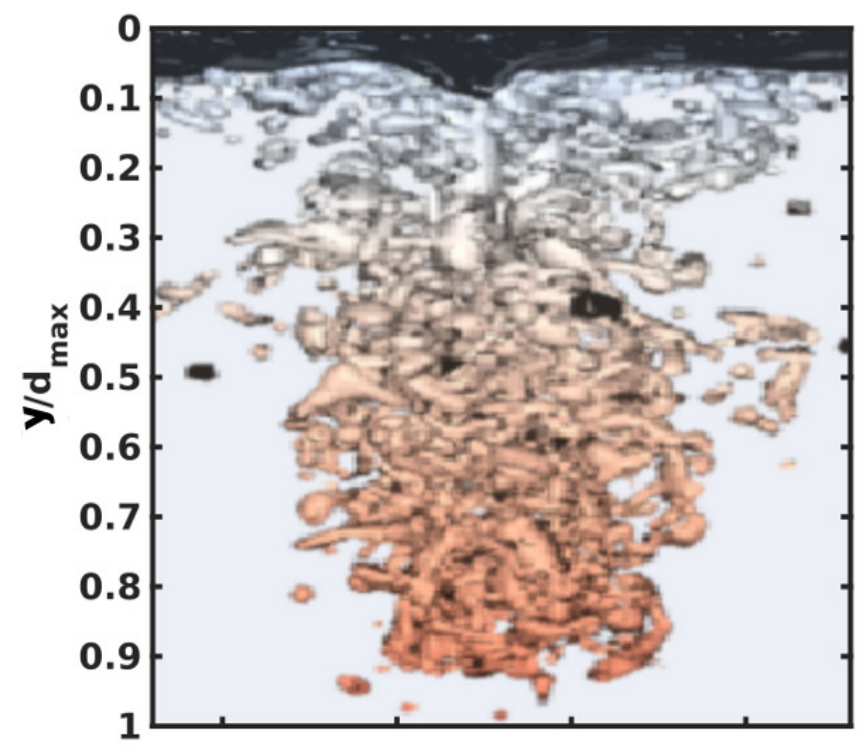

(b)

Figure 6: (a) Experimental snapshot of the bubble cluster below the liquid pool with points of the interface obtained from numerical simulation (b) Numerically obtained bubble cluster at $F r_{D} F r_{L}=1.4$; both experimental snap and numerical contours are plotted at $\mathrm{T}=300$ starting from jet touching the pool. 

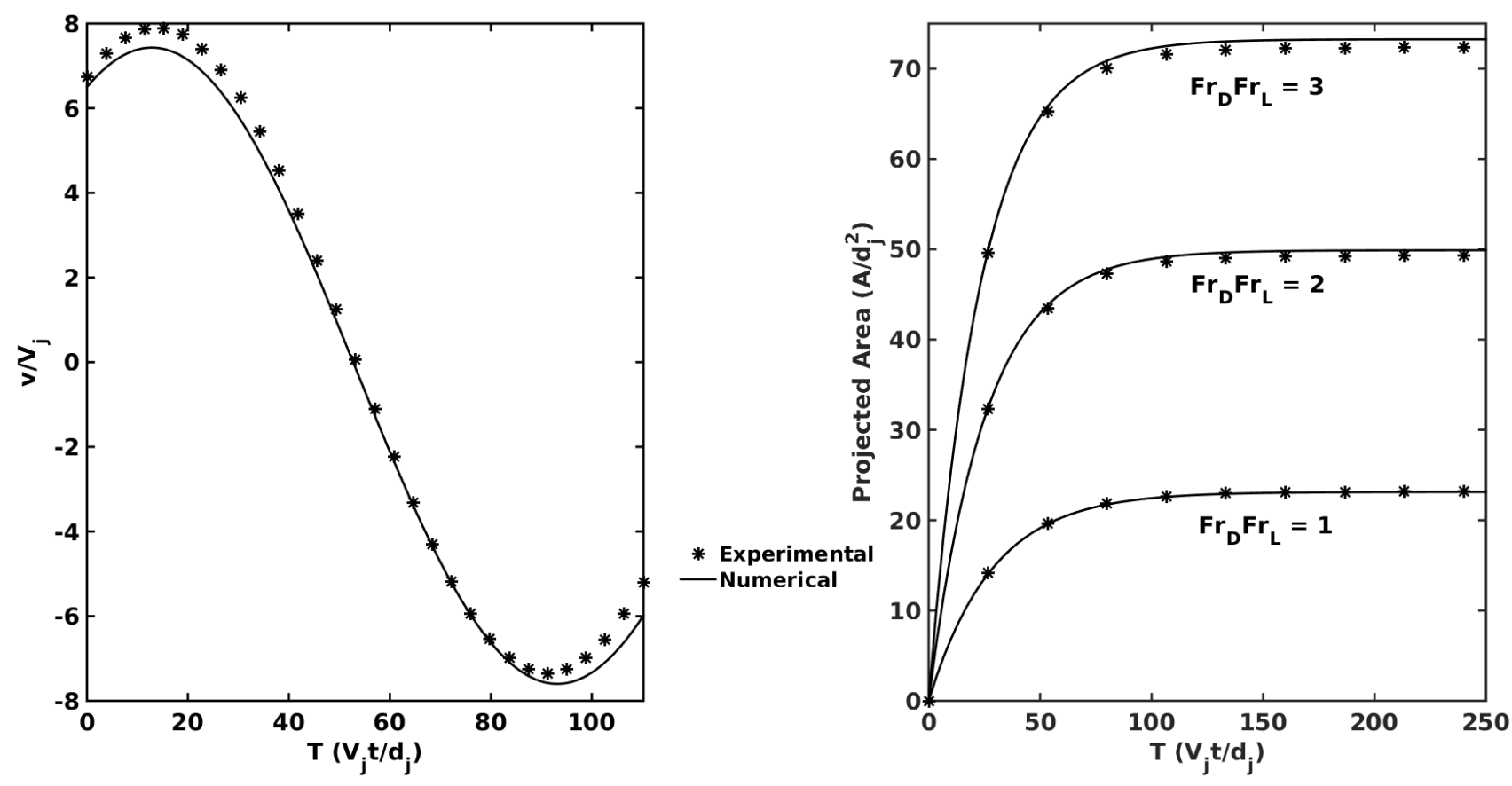

Figure 7: Comparison between experimental and numerically obtained results for temporal variation of (a) Velocity vector of a typical bubble in the entrained region at $F r_{D} F r_{L}=1.8$ (b) Projected area of the entrained bubble cluster

its identity (with lower velocity than expected due to impact with the pool) and after the pinch off of the first bubble, jet continues to entrain bubbles inside the pool. Finally, location 4 is in the liquid region of the first annular bubble that pinches off. Figure 5 clearly demonstrates that the velocity profile is independent of spatial refinement beyond case 4 , justifying our approach to select the mesh parameters of case 5 as our fundamental configuration. Next, the numerical code employed is tested for its validity by virtue of its correspondence with the experimental observations. Figure 6 contains the results of validation test carried out for $F r_{D} F r_{L}=1.4$. The numerically simulated interface structure shows a remarkable similarity with the experimentally obtained bubble cluster interface. Velocity variation of a bubble in the cluster with time is obtained from experimental snaps and reported in Figure 7(a) along with data of a bubble from numerical simulation. A close match between numerical prediction and experimental observation validates the developed model. Moreover, efforts are also made to measure the area occupied by the bubble cluster from temporal snaps of experimental observation. Development of cluster area with time from experiment is plotted in Figure 7(b) along with numerical findings. It can be observed from the figure that experiment and numerical simulation corroborates similar observations for a range of $F r_{D} F r_{L}$. Next section illustrates the two possible outcomes of the 


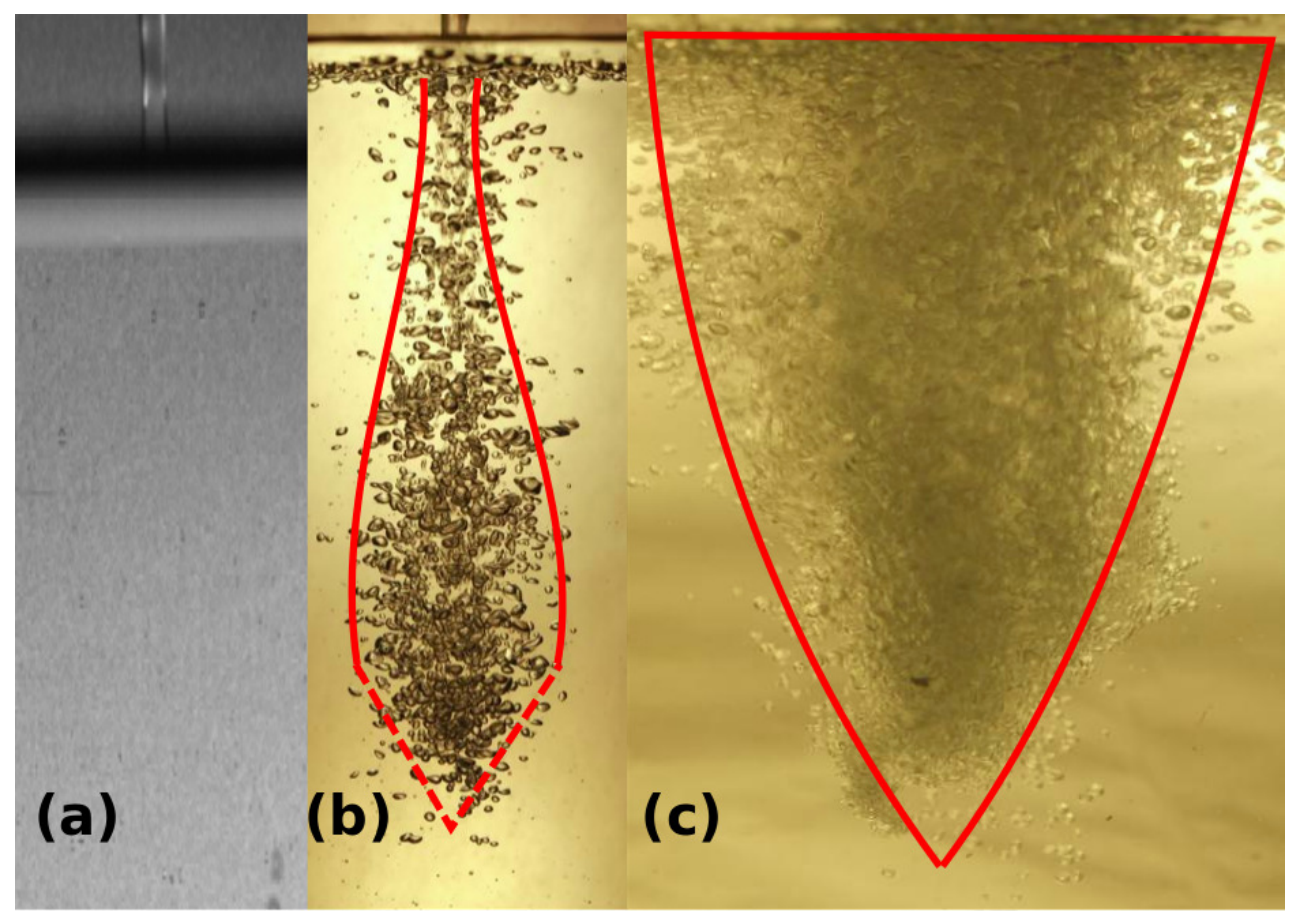

Figure 8: Possible outcomes as the jet impinges onto the pool: (a) No entrainment $F r_{D} F r_{L}=$ 0.125, (b) Continuous Entrainment with diverging converging cluster $F r_{D} F r_{L}=1.25$ and (c) Continuous Entrainment with triangular entrainment region $F r_{D} F r_{L}=3.8$

jet-pool interactions, no entrainment and vigorous bubble cluster formation. These are obtained from experimental snapshots.

\section{Continuous and No Entrainment}

Figure 8 shows two types of possibilities when the liquid jet impinges the water surface. Even though, the inception of air bubble entrainment is not easily characterized.Experimental observation showed that for a low inertial jet, the flow is likely to be laminar and there is no air entrainment (Figure 8(a)). With increase in $F r_{D} F r_{L}$, an air bubble cluster is observed below the impact point inside the test pool. In Figure 8(b), at $F r_{D} F r_{L}=1.1$, we have showed the pattern of air entrainment which is diverging-converging in shape. At even higher $F r_{D} F r_{L}$, a triangular entrainment region is observed as shown in Figure 8(c). The outer bound of the bubble cluster is shown in Figure with red line. Depth of the cluster is observed to be varied as a function of jet inertia as discussed later on. The transition between entrainment and no entrainment is not well defined and is prompt $\frac{30 \mid 37}{3}$. But it has been observed (discussed later) that entrainment height diminishes with $F r_{D} F r_{L}$ and there exist a critical $F r_{D} F r_{L}$ below which entrainment is 


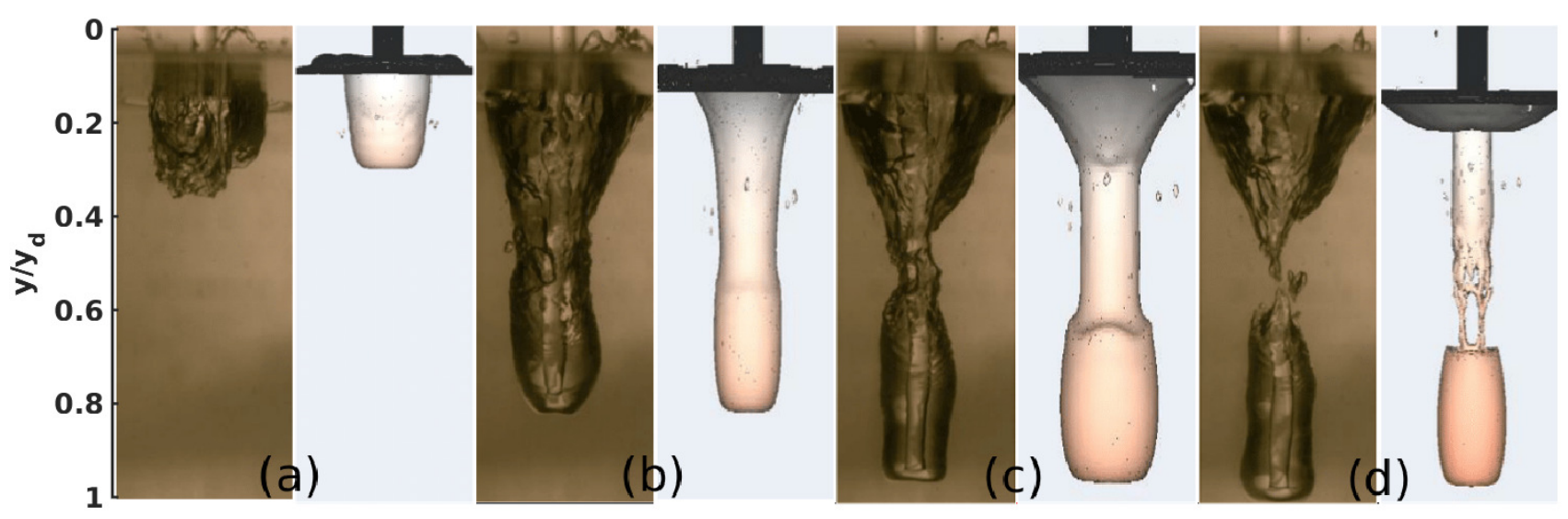

Figure 9: The temporal record of the onset of entrainment; (left: experimental and right: numerical) (a) Jet impacts the liquid pool and forms a cavity at $T=\frac{V_{j} t}{d_{j}}=1$, (b) Elongation of the cavity formed at $T=1.95$, (c) Necking of the elongated cavity at $T=2.5$ as surface tension force tries to overcome jet's inertia and (d) Pinch off of the first annular air bubble at $T=3.5$. $\left(F r_{D} F r_{L}=2.2\right)$.

not present. Assessment of this transition exactly in terms of $F r_{D} F r_{L}$ is quite difficult which requires precise control of flow rate. Our present experimental setup was not equipped for such precision and therefore we have focused on the entrainment regime. But Harby et al. ${ }^{30}$ have defined inception of entrainment as entry of more than three bubbles in liquid pool in three minutes and proposed inception velocity as function of $\frac{l_{j}}{d_{j}}$. Our isolated efforts showing no entrainment

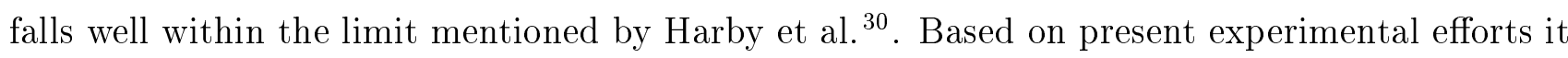
can be ensured that for $F r_{D} F r_{L}$ less than 0.1 will not show any entrainment. For precise limit one may follow limits proposed by Harby et al. .30 . Next, we present a subtle account of the onset of entrainment process.

\section{Onset of Entrainment}

To understand the two opposite possible outcomes mentioned above, we tried to observe the onset of entrainment. Onset will be propagated or suppressed to reach either in the no entrainment or continuous entrainment scenario, respectively. Figure 9 consists of a detailed account of the jet pool interaction from the point of impact to the point of pinch off. Both experimental snapshots and numerical tracer contours are shown side by side to establish the onset dynamics. As the jet strikes the liquid pool, a cavity is formed with the liquid core at the centerline surrounded by the air medium which by virtue of inertia penetrates inside resulting in formation of a long cylinder. 


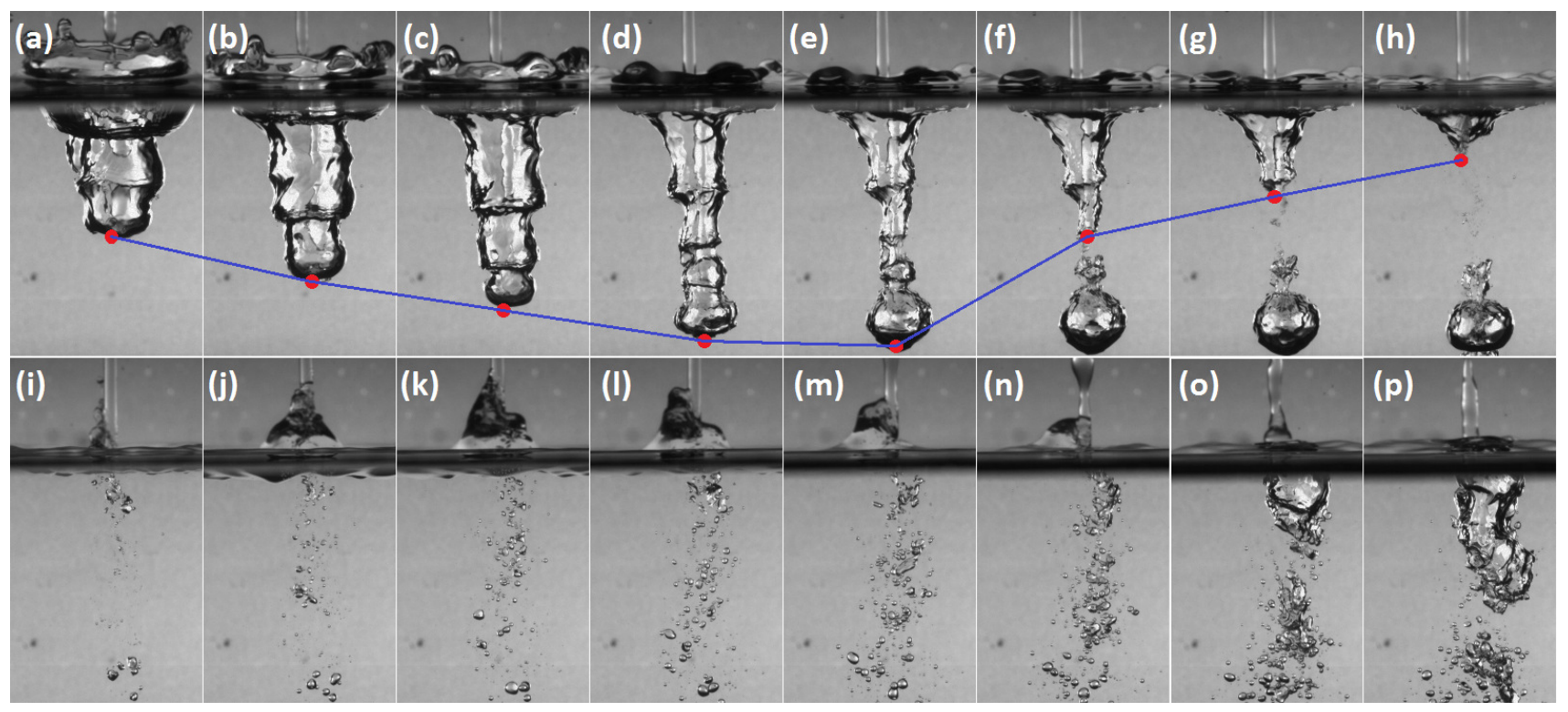

Figure 10: Experimental snapshots of pinch off for $F r_{D} F r_{L}=1$ at $T=$ (a) 2.04, (b) 2.28, (c) 2.52, (d) 2.76, (e) 2.88, (f) 3, (g) 3.24, (h) 3.35, (i) 3.5, (j) 3.75, (k) 4, (l) 4.25, (m) 4.5, (n) 5, (o) 5.5 and (p) 6

Surface tension and circulation surrounding the jet (as discussed later) results in necking which propagates further and pinches off as shown in Figure 9(d). The process has been studied from high speed images in detail for the onset of entrainment for $F r_{D} F r_{L}=1$ (Figure 10). It can be observed from experiments that upon striking the free surface, due to inertia, jet creates a dimple and propagates downwards to form a cavity filled with air (Figure 10(a)). With time, the cavity enlarges (Figure 10(b) - 10(e)) before surface tension becomes significant and starts contracting the cavity back to its original free surface. In the initial period, air cavity is formed as stepped cylinder with continuously decreasing diameter (Figure 10(a) - 10(c)). At a critical depth, surface tension starts dominating and cylinder turns into sphere (Figure 10(d)) to initiate the contraction of the cavity. At this level, inertia gets weakened and surface tension starts dominating. Due to conversion of cylindrical cavity into spherical air mass (Figure 10(e)), pressure waves are generated which propagates in upward direction and contracts the stepped cylinder towards the jet (Figure 10(e) - 10(f)), prompting collapse of the cavity. While the cavity is being collapsed, spherical air mass at the bottom of the cavity forms neck (Figure 10(e)) as a result of higher amplitude of three dimensional pressure waves. This leads towards pinching (Figure 10(f)) off the spherical bubble along with some satellites and retraction of rest cavity towards the free surface (Figure 10(f) - 10(h)). Experiment shows that collapse of cavity generates an upward moving liquid jet (Figure 10(i)) thicker than the impinging one (Figure 10(j)). These two counteracting 
(a)

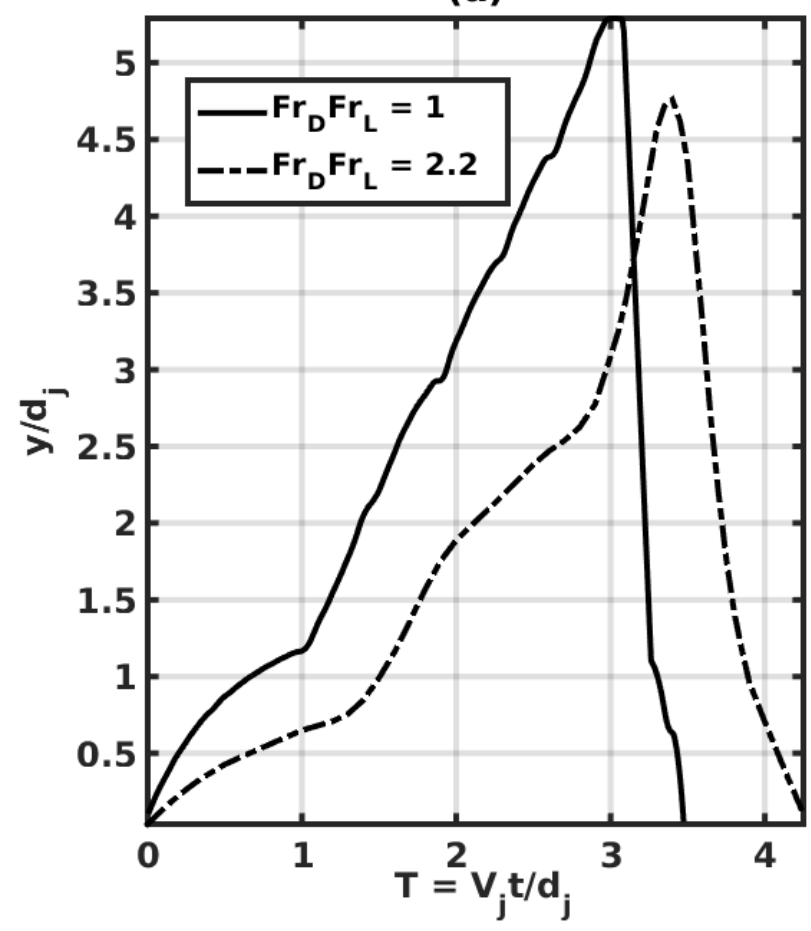

(b)

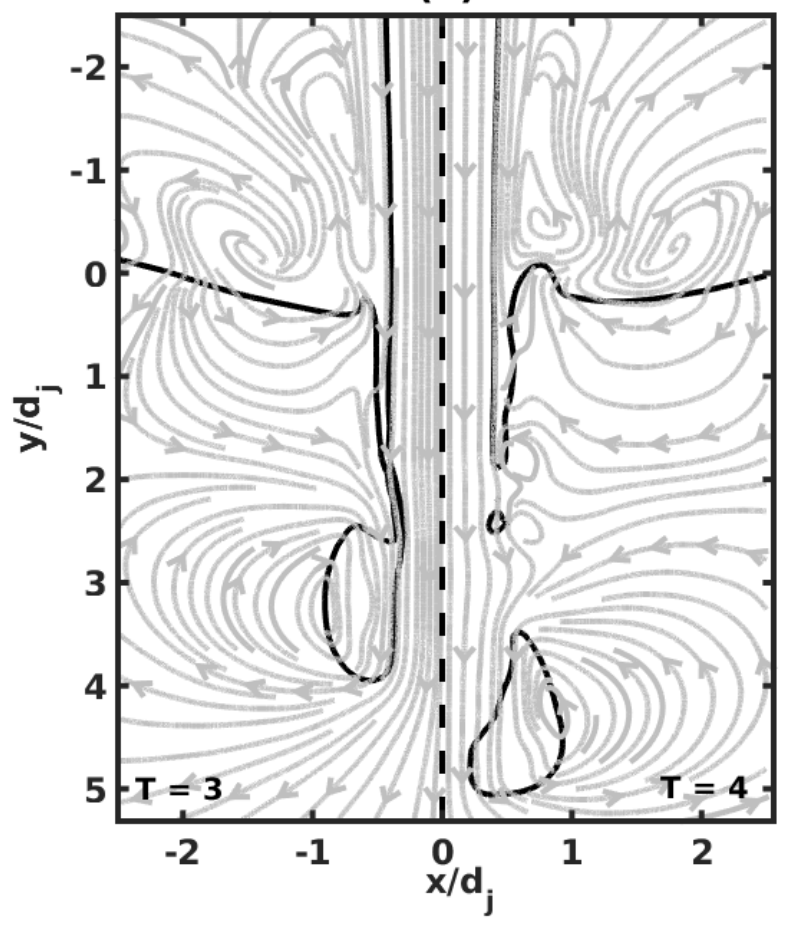

Figure 11: (a) Depth of penetration of liquid cavity formed during the impact of jet onto the pool and subsequent collapse of cavity. (b) Streamlines developed around the formed cavity before (on left) and after (on right) pinch off.

jets entrap air bubble in between them (Figure $10(\mathrm{k})$ ). As the velocity of impinging jet is higher than the pressure wave jet, entrapped bubbles move down in the pool (Figure 10(k) $10(1))$. With time, jet created by pressure wave falls down (Figure $10(\mathrm{~m})-10(\mathrm{n})$ ) and impinging jet starts second cycle of cavity formation (Figure $10(0)$ ). The whole process repeats many a times to entrap more and more bubbles (Figure $10(\mathrm{p})$ ) and finally form a cluster as shown in experimental observation of Figure 8(c). The detached spherical bubble in each cycle comes back to free surface and in its path it dislodges daughters due to impact from bubbles in cluster. At lower product of Froude numbers $\left(F r_{D} F r_{L}\right)$, inertia never becomes dominated over surface tension and gravitational collapse strength to show all these sequences for formation of bubbly cluster. Impingement of jet at lower $F r_{L} F r_{D}$, creates a cavity but its propagation in downward direction is suppressed and immediate collapse causes no entrainment of bubbles. But this requires further proof in future efforts.

One can clearly understand from the high speed image sequences of Figure 10 that during onset, the cavity collapses (surface tension driven) at a faster rate than formation (inertia dominated). Figure 11(a) shows the maximum depth of the cavity with time as obtained from numerical 


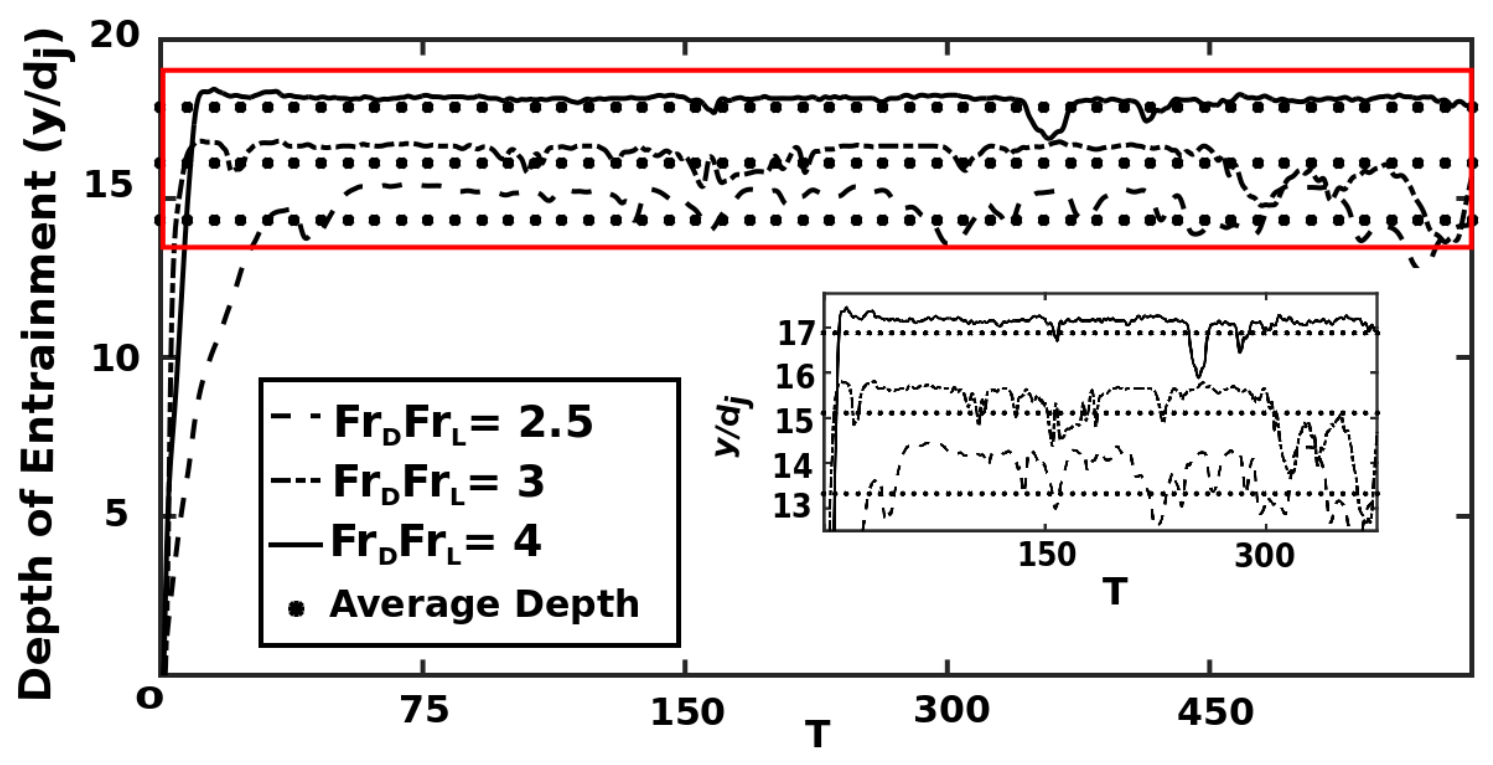

Figure 12: Temporal variation of maximum depth of entrainment. The inset figure takes a closer look at the variation of the height of entrainment, which pertains to a nearly constant value.

simulations, for a complete cavity cycle. Initially depth increases at a slower rate till pinch off of the bubble, as represented by steep fall in depth. Subsequently, collapse of cavity occurs at a faster rate as shown in Figure 11(a). With the streamline field in Figure 11(b) obtained through numerical simulations, we have attempted to study the dynamic process of pinch off. As the cylindrical cavity elongates (by virtue of jet's inertia) the surrounding air-sheathe gets thinner and a region of high circulation is realized around the jet. As the jet tries to further elongate the cavity against the resistance of the pool, the developing circulations catapult the bubble and pinches it off from the thin air sheathe. A look at the streamlines near the pool surface justifies the movement of the point of pinch off towards the incoming jet as the cavity collapses back. As the cycle repeats, more bubbles are entrapped which ultimately leads to the formation of bubble cluster below the surface. Next, we illustrate the dynamic nature of the generated bubble cluster and characterize it based on the strength of the jet.

\section{Dynamics of Bubble Cluster}

Once the cluster of different sized, shaped and interacting bubbles comes into existence, dynamics of an individual bubble is hard to follow. Over all nature of the bubble cluster remains almost same and the depth of entrainment attains a steady state value. In Figure 12, from 


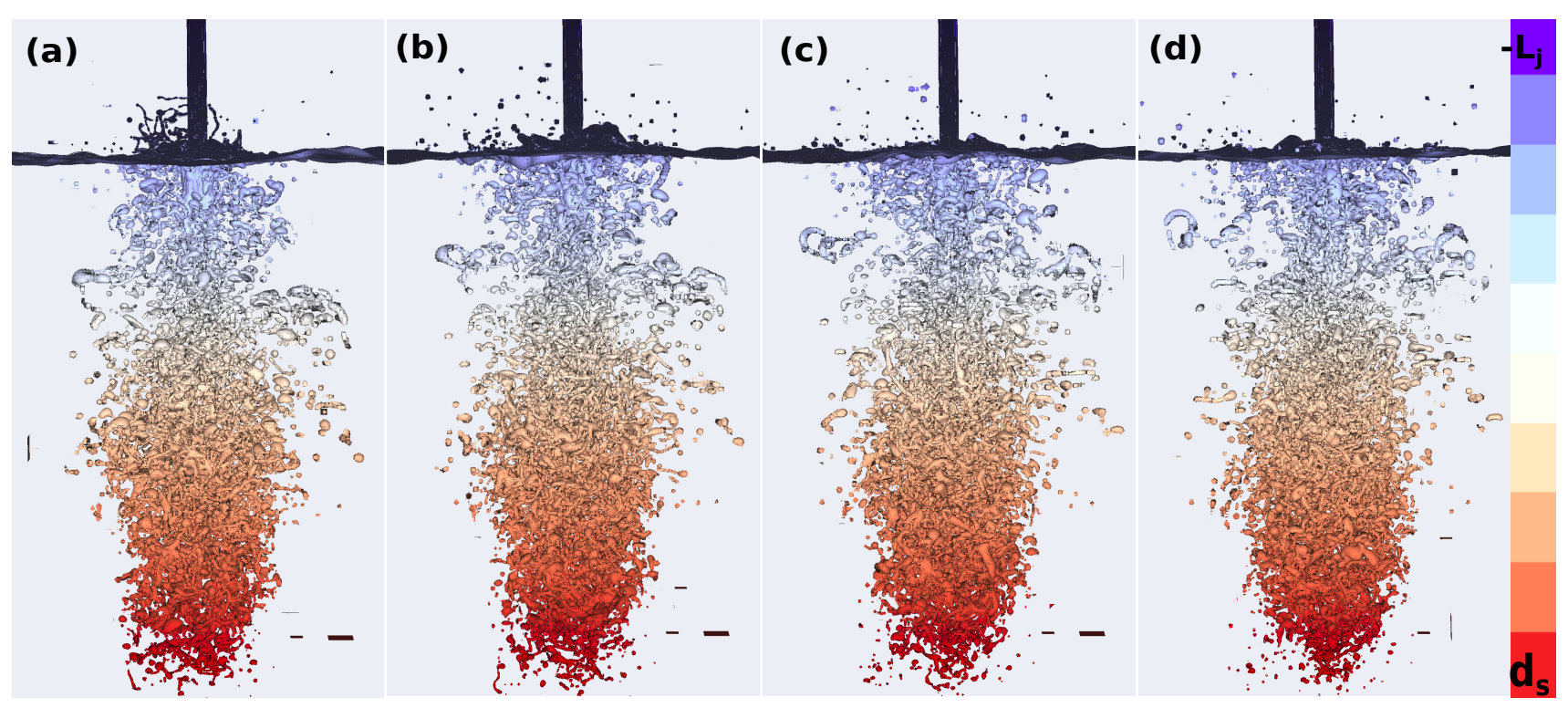

Figure 13: Attainment of steady state entrainment height for $F r_{D} F r_{L}=4$ with the interface colored by the depth as measured from the liquid pool interface at $T=$ (a) 85, (b) 90, (c) 95 and (d) 100

experimental observations, we have shown the variation of the depth of entrainment with time for different strengths $\left(F r_{D} F r_{L}\right)$ of the jet. Experimentally, after onset (increasing depth with time), saturation of depth is observed up-to a prolonged period. For different jet strengths, we have also plotted the average depth (with dots) which is in the range of saturation. It shows that after onset, depth of entrainment is not a function of time, rather depends on the strength of the impinging jet. This nature has been shown clearly in inset of Figure 12 .

Steady penetration of entrainment inside pool is also confirmed from numerical simulations. In Figure 13, detailed Volume of Fluid tracer colored using the distance from the liquid pool interface as the scalar has been shown. Numerical simulation shows that the depth of penetration of the bubble cluster pertains to a constant value as the time passes. Present numerical simulation matches well with experimental observation here. In Figure 12, experimentally, we also observed increase of steady depth for three $F r_{D} F r_{L}$. Experimental and numerical snapshots of entrainment pattern at different $F r_{D} F r_{L}$ are shown in Figure 14 to confirm the story of interface locations. From these photographic observation and numerical phase contours we obtain that entrained air penetration depth is proportional to $F r_{D} F r_{L}$. A complete range of depth $\left(d_{s}\right)$ variation of entrainment from free surface for a wide range of $F r_{D} F r_{L}$ is shown in figure 15. Both experimental and numerical data points are shown in Figure 15. To express this increasing pattern of cluster depth as a function of $F r_{D} F r_{L}$, we propose correlations (equation 8 


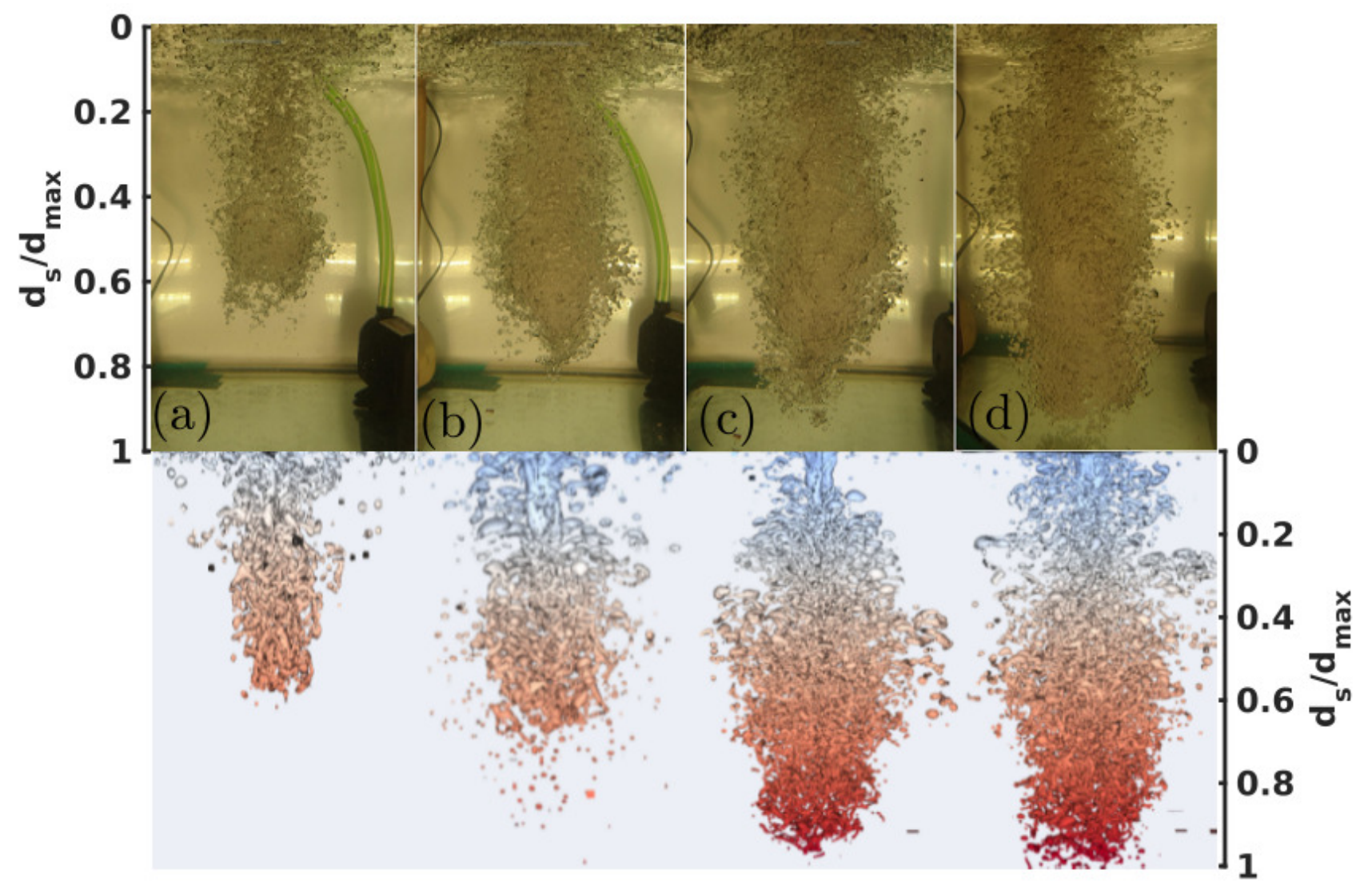

Figure 14: Steady entrainment pattern for $F r_{D} F r_{L}=$ (a) 1.4, (b) 2, (c) 3.25 and (d) 3.75

for $\operatorname{Fr}_{D} F r_{L}<1.2$ and equation 9 for $\left.F r_{D} F r_{L}<1.4\right)$ with two empirical constants each in the form of a power law. The change in behavior of the curves is because of the difference in entrainment cluster regimes as observed in Figure 8. The empirical constants are fitted from experimental observations with Summed Square of Residue (SSE) equal to $7.5 \mathrm{X} 10^{-2}$ and $5 \mathrm{X}$ $10^{-2}$ and R-square value as 0.9 and 0.95 respectively for equation 8 and 9 .

$$
\begin{gathered}
\frac{d s}{d j}=9.45\left(F r_{D} F r_{L}\right)^{0.33} \\
\frac{d s}{d j}=3.3\left(F r_{D} F r_{L}\right)^{1.2}
\end{gathered}
$$

To show the predictability of already available correlations against our experimental observations and establishment the improvement in prediction using equation (8), power law as proposed by Ohkawa et al. (1986) ${ }^{\frac{38}{38}}$ is also shown in Figure 15 . Further, volumetric strength of bubble cluster has been also observed to change with jet strength $\left(F r_{D} F r_{L}\right)$ in Figure 14 . We have estimated the variation of the projected area of the cluster in a two dimensional photo- 


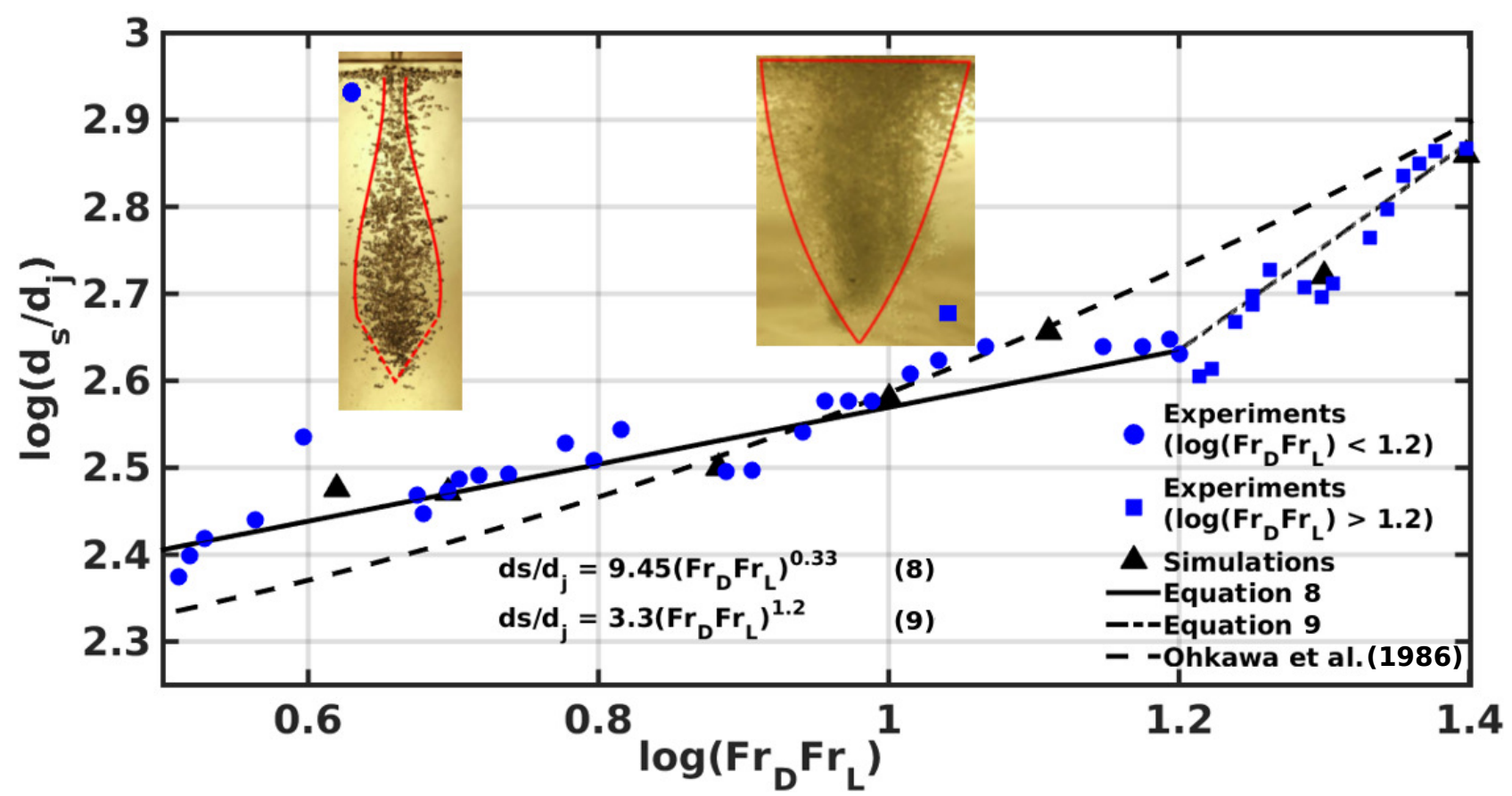

Figure 15: Steady state depth of entrainment as a function of the product of Froude numbers, $\mathrm{Fr}_{D} \mathrm{Fr}_{L}$; both experimental and numerical data points are mentioned.

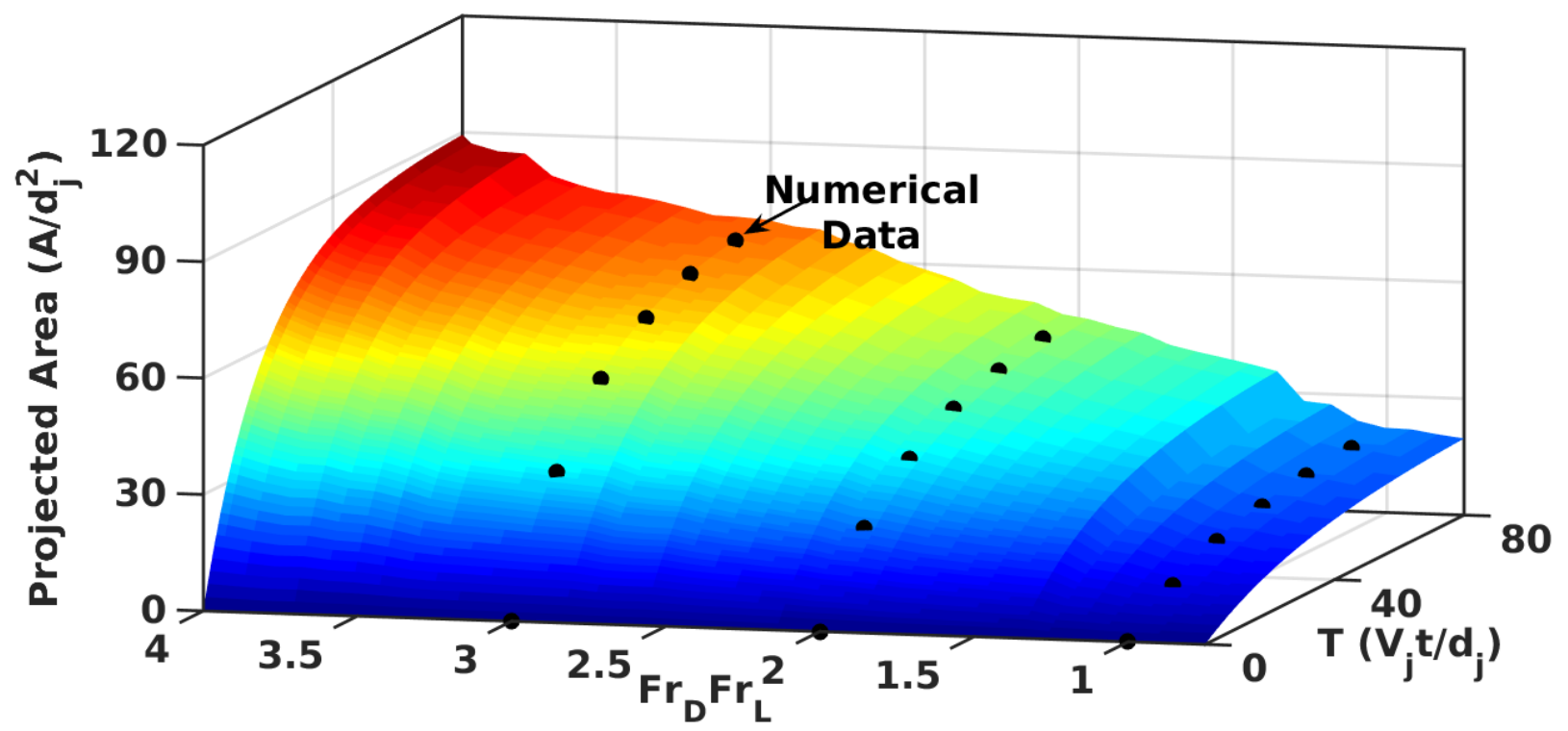

Figure 16: Projected area of the region affected by entrained bubble cluster; plane is constructed by experimental observations and numerical data points are shown by symbols. 


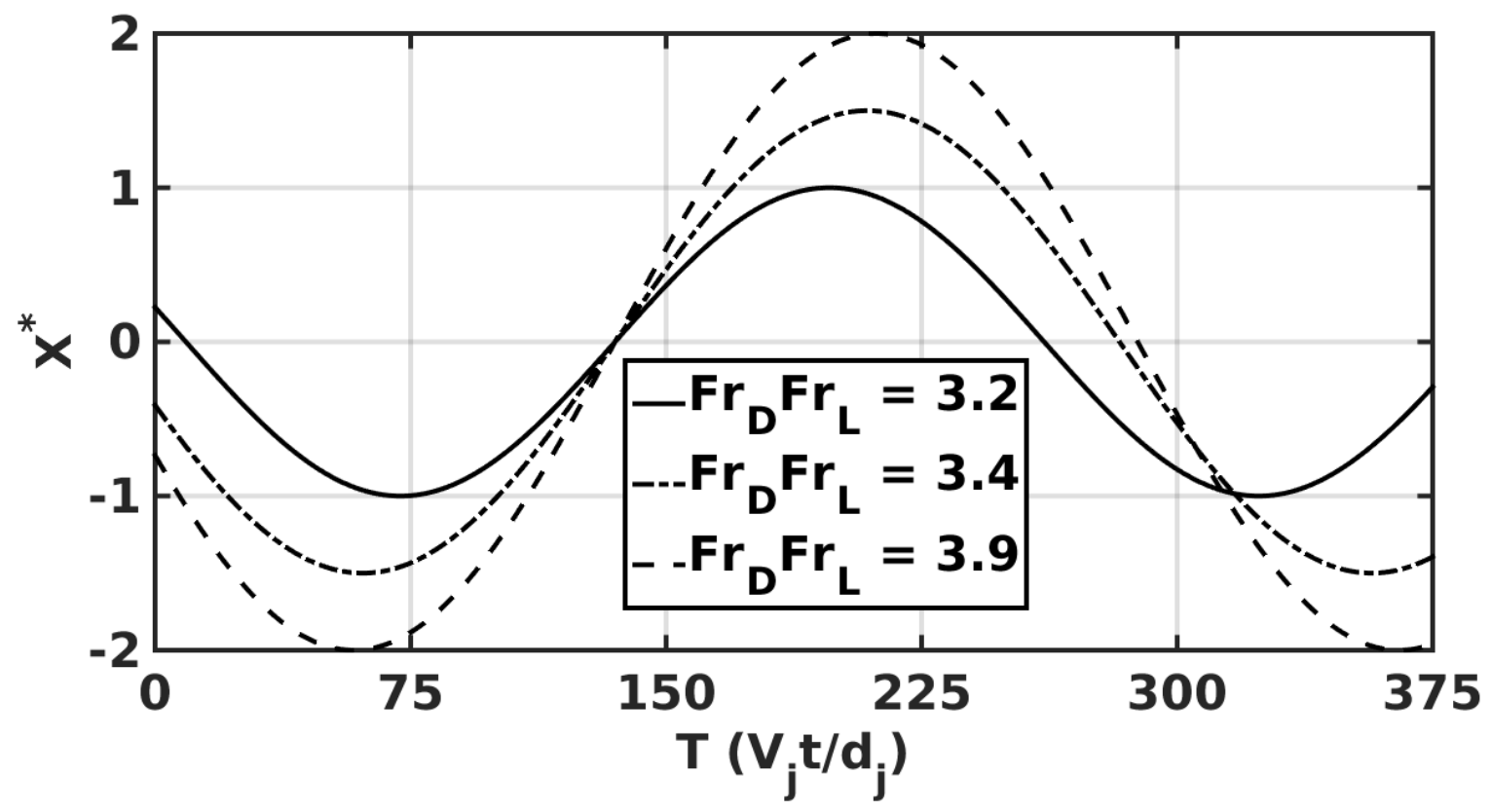

Figure 17: Temporal variation of the normalized abscissa of the centroid related to the entrainment cluster.

graphic plane along with time at different jet strength. In Figure 16, projected area is plotted as a function of $\left(F r_{D} F r_{L}\right)$ and time after post processing our experimental data. One can clearly observe from this Figure that the projected area increases with $F r_{D} F r_{L}$ and time. Variation of projected area with time can be attributed to development of cluster initiating from onset. On the other hand, strength of inertia can be clearly seen from higher projected area at strong jet than a weak one.

From close look at experimental snaps, it has been observed that the centroid of the projected area (location $\mathrm{x}$ ) shows periodic oscillation. Binary two dimensional images, as shown in Figure 1(b), are used to calculate the location of the centroid. Equation 10 is followed for calculation, where $\bar{x}\left(x_{i}, y_{i}\right)$ is the location of the pixel in the binary image corresponding to the cluster area (non-zero value) and $\mathrm{N}$ is the total number of non-zero pixels.

$$
X_{\text {centroid }}=\frac{\int \bar{x} d A}{A}=\frac{\sum_{i=1}^{N} \bar{x}_{i}}{N}
$$

Upon non-dimensionalizing with its mean and standard deviation as $X^{*}=\left(\frac{x-m e a n(x)}{\sigma_{x}}\right)$, we obtained the periodic movement as illustrated in equation 11 .

$$
\frac{\partial^{2} X^{*}}{\partial t^{2}}+\omega^{2} X^{*}=0
$$




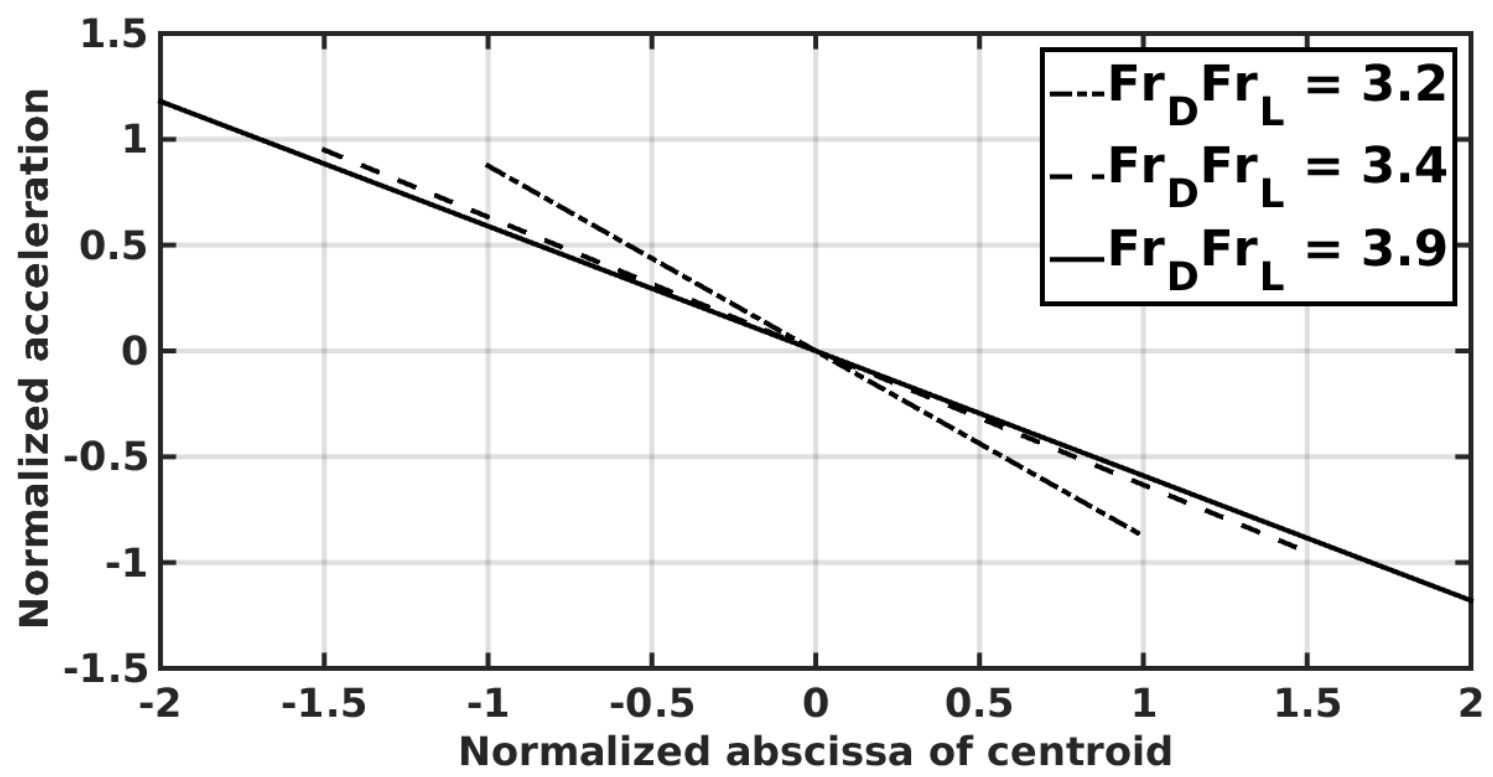

Figure 18: Variation of the acceleration of the centroid with its normalized abscissa related to the entrainment cluster.

Oscillations are observed in phases at all azimuthal planes. This represents the pseudo randomness of the bubble cluster configuration having constant depth over time. As cluster is constructed by pinch off of bubbles from free surface, temporal dependence is not eliminated and became prompt in lateral direction. From experimental observation, variation of $X^{*}$ is obtained with non-dimensional time $\left(T=V_{j} t / d_{j}\right)$ and plotted in Figure 17 . The periodic nature is clearly depicted in Figure 17 having higher amplitude for lower $F r_{D} F r_{L}$. Increase of $\omega$ for lower $F r_{D} F r_{L}$ can also be seen from this figure as oscillation time periods for $F r_{D} F r_{L}=3.2$ is lower than $F r_{D} F r_{L}=3.9$. In equation 11, acceleration of centroid for the bubble cluster varies proportionally with its shift from the longitudinal jet plane. To depict that clearly, in Figure 18 , we showed normalized acceleration for different experimental conditions of $F r_{D} F r_{L}$. In all these cases, linear patterns have been observed confirming validity of equation 11. At higher $F r_{D} F r_{L}$, it has been observed that the centroid acceleration increases and it deviates more from the jet plane. We propose that acceleration of the centroid increases at a faster rate with shift from jet plane for lower $\mathrm{Fr}_{D} F r_{L}$ (resulting in higher proportionality constant $\omega^{2}$ in equation 11).

Present experimental effort establishes steady over all behavior of the cluster but keeps provision for monitoring local behavior of bubbles. Next section targets towards understanding of individual bubble dynamics with the help of visualization and signals from electrical conductivity probe. 


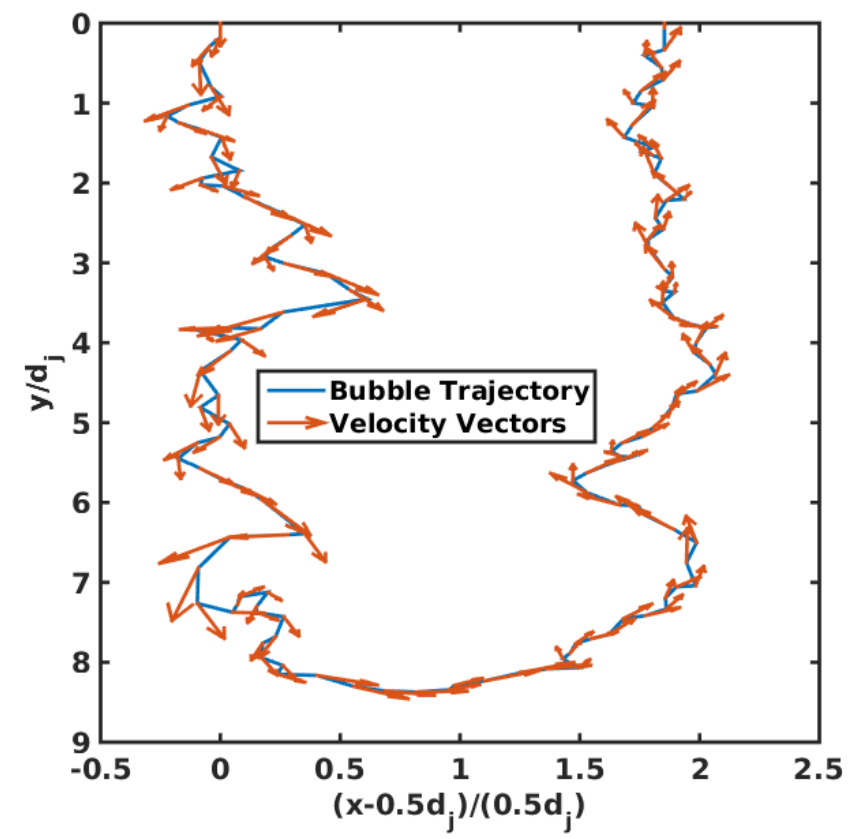

Figure 19: Bubble trajectory in the entrained region at $F r_{D} F r_{L}=1.8$, as obtained from experimental observation. The bubble inception occurs at the tip of the thin air sheathe, traverses through the entrainment region, stops the downward motion as the buoyancy dominates its motion and erupts back at the free surface.

\section{Bubble Kinematics}

The bubble cluster consists different sized bubbles consistently making and breaking within the triangular entrainment region. The trajectory of a bubble is followed at a comparatively low speed entrainment phenomenon from photographic observation in experiments and represented in Figure 19. After birth, the bubble moves downwards inside the cluster and reaches the maximum depth. It halts there for sometime before coming up from the other side of the cluster. Its downward movement is driven by inertia and upward movement is due to buoyancy pull. We have also shown instantaneous velocity in the plot. In between initiation and collapse, the bubble is dominated by inertia, interaction and buoyancy pull inside the cluster (Figure 19).

Electrical conductivity probe signals have been recorded for varying flow rates and different lengths of jets inside the bubble entrainment zone. The physical illustration of voltage signal is shown in Figure 2. The sharp downfalls represent the presence of bubbles. Representative signal of probes in experimental cluster is shown in inset of Figure 20, Even though the voltage signal, which varies in between 3.5 to 1.5 volts, clearly depicts the presence of entrapped air bubbles, it is difficult to get any kinematic inference. Therefore, from raw electrical signals, probability density function (PDF) has been plotted for three representative flow-rates in Figure 20. The 


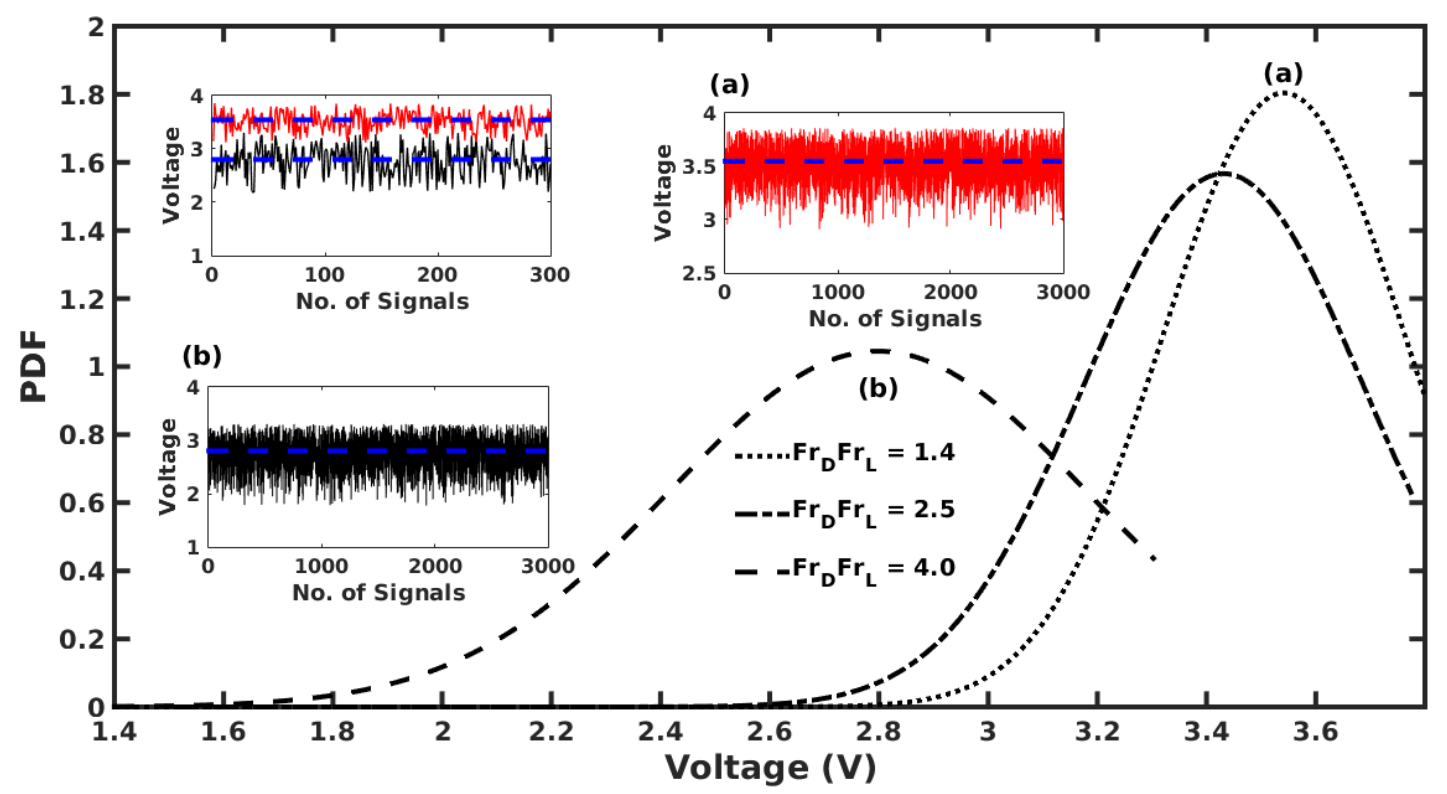

Figure 20: Characteristic probability density function of the experimental probe signals at different values of $\mathrm{Fr}_{D} F r_{L}$. More the peak is shifted towards the left, the high density of bubbles are present at the spatial location of the electrodes.

peak in PDF shifts to the left and lowers down as the flow rate increases. Probability of getting lower voltage signifies vigorous cluster characteristics and high probability of bubble presence at a given spatial coordinate. With increase in jet flow-rate, entrainment strength increases and causes peak at lower voltage. This shows more and more bubbles are being trapped due to its random presence in the cluster at higher flow rates. This clearly demonstrates the bubble kinematics as well as cluster vibrations. Signals of probe confirm the presence of bubble in the cluster and its peak of PDF at lower value signifies higher mobility of bubbles at the cluster. At lower jet speed bubbles are produced but exhibit comparative calm behavior to have liquid contact at the probe tip. On the other hand, at high jet speed, random motion of the generated bubble keeps always gaseous phase in contact with probe. Present observation establishes highly random kinematic behavior at high jet speed.

\section{Conclusion}

Impingement of water jet on a pool is studied using full-scaled experiments and direct numerical simulation. High speed imaging and electrical conductivity probes are used in experimental instrumentation. Depending on jet's strength, two opposite regimes have been identified as 
no entrainment and conical bubble cluster. Formation of inertia dominated cavity and surface tension dominated collapse have been identified as the basic consequences behind the onset of bubble entrainment as a cluster. Repetitive formation and breakage of air cavity in the pool results in disconnected but interacting bubble population. Mutual interplay between inertia and surface tension determines the depth of entrained region which has been reported as a function of the product of Froude Numbers $\left(F r_{D} F r_{L}\right)$ in two correlations for different regimes with boundary at $F r_{D} F r_{L}=1.2$. Proposed correlation shows accurate prediction (R-squared of 0.9 and 0.95 ) of depth for a wide range of jet diameters and heights. Using high speed images, increasing projected area of entrainment has been observed for higher $\operatorname{Fr}_{D} F r_{L}$ and time which establishes the affected zone during entrainment. Kinematics of entrainment has been studied using spatio-temporal variation of centroid location of the cluster. Acceleration of the centroid is found to be linearly varying with deviation of the entrained region from the plane of jet impact. Trajectory of a single bubble has been also reported in the study from visualization to establish formation, downward traverse, stabilized floating at critical height, buoyancy driven approach towards free surface and collapse in its whole life cycle.

\section{Literature Cited}

1. Sanjay V, Das AK. On air entrainment in a water pool by impingement of a jet. AIChE Journal;63(11):5169-5181.

2. Evans GM, Jameson GJ, Rielly CD. Free jet expansion and gas entrainment characteristics of a plunging liquid jet. Experimental Thermal and Fluid Science. 1996;12(2):142-149.

3. Biń AK. Gas entrainment by plunging liquid jets. Chemical Engineering Science. 1993; 48(21):3585-3630.

4. Sene KJ. Air entrainment by plunging jets. Chemical Engineering Science. 1988;43(10):26152623.

5. Goldring BT, Mawer WT, Thomas N. Level surges in the circulating water downshaft of large generating stations. In: Proc. 3rd Intl Conf. on Pressure Surges. 1980; pp. 279-300. 
6. Bonetto F, Lahey RT. An experimental study on air carryunder due to a plunging liquid jet. International Journal of Multiphase Flow. 1993;19(2):281-294.

7. McKeogh EJ, Ervine DA. Air entrainment rate and diffusion pattern of plunging liquid jets. Chemical Engineering Science. 1981;36(7):1161-1172.

8. Van de Donk JAC. Water aeration with plunging jets. TU Delft, Delft University of Technology. 1981.

9. El Hammoumi M, Achard JL, Davoust L. Measurements of air entrainment by vertical plunging liquid jets. Experiments in fluids. 2002;32(6):624-638.

10. Chanson H. Air bubble entrainment in free-surface turbulent shear flows. Academic Press. 1996.

11. Oguz HN, Prosperetti A, Lezzi AM. Examples of air-entraining flows. Physics of Fluids A: Fluid Dynamics (1989-1993). 1992;4(4):649-651.

12. Lin TJ, Donnelly HG. Gas bubble entrainment by plunging laminar liquid jets. AIChE Journal. 1966;12(3):563-571.

13. Bin AK. Gas entrainment by plunging liquid jets. Forschung im Ingenieurwesen. 1988; 54(4):136-136.

14. Fetisov YM. Experimental investigations of the conditions of the onset of air entrainment by plunging liquid jets. Power Technology and Engineering (formerly Hydrotechnical Construction). 1996;30(2):87-91.

15. Cummings PD, Chanson H. Individual Air Bubble Entrapment at a Planar Plunging Jet With Near-Inception Flow Conditions. In: Proceedings, 13th Australasian Fluid Mechanics Conference. 1998; pp. 13-18.

16. Chanson H, Cummings PD. An experimental study on air carryunder due to a plunging liquid jet. International journal of multiphase flow. 1994;20(3):667-670.

17. Cummings PD, Chanson H. An experimental study of individual air bubble entrainment at a planar plunging jet. Chemical Engineering Research and Design. 1999;77(2):159-164. 
18. Soh WK, Khoo BC, Yuen WYD. The entrainment of air by water jet impinging on a free surface. Experiments in fluids. 2005;39(3):498-506.

19. Chanson H, Aoki Si, Hoque A. Physical modelling and similitude of air bubble entrainment at vertical circular plunging jets. Chemical engineering science. 2004;59(4):747-758.

20. Clanet C, Lasheras JC. Depth of penetration of bubbles entrained by a plunging water jet. Physics of Fluids (1994-present). 1997;9(7):1864-1866.

21. Van de Sande E, Smith JM. Jet break-up and air entrainment by low velocity turbulent water jets. Chemical Engineering Science. 1976;31(3):219-224.

22. Qu XL, Khezzar L, Danciu D, Labois M, Lakehal D. Characterization of plunging liquid jets: A combined experimental and numerical investigation. International Journal of Multiphase Flow. 2011;37(7):722-731.

23. Chen F, Hagen H. A Survey of Interface Tracking Methods in Multi-phase Fluid Visualization. In: Visualization of Large and Unstructured Data Sets - Applications in Geospatial Planning, Modeling and Engineering (IRTG 1131 Workshop), edited by Middel A, Scheler I, Hagen H, vol. 19 of OpenAccess Series in Informatics (OASIcs). Dagstuhl, Germany: Schloss Dagstuhl-Leibniz-Zentrum fuer Informatik. 2011; pp. 11-19.

URL http://drops.dagstuhl.de/opus/volltexte/2011/3091

24. Deshpande SS, Trujillo MF. Distinguishing features of shallow angle plunging jets. Physics of Fluids (1994-present). 2013;25(8):082103.

25. Brouilliot D, Lubin P. Numerical simulations of air entrainment in a plunging jet of liquid. Journal of Fluids and Structures. 2013;43:428-440.

26. Durve AP, Patwardhan AW. Numerical and experimental investigation of onset of gas entrainment phenomenon. Chemical engineering science. 2012;73:140-150.

27. Zhu Y, Oguz HN, Prosperetti A. On the mechanism of air entrainment by liquid jets at a free surface. Journal of Fluid Mechanics. 2000;404:151-177.

28. Kersten B, Ohl CD, Prosperetti A. Transient impact of a liquid column on a miscible liquid surface. Physics of Fluids (1994-present). 2003;15(3):821-824. 
29. Belden J, Ravela S, Truscott TT, Techet AH. Three-dimensional bubble field resolution using synthetic aperture imaging: application to a plunging jet. Experiments in fluids. 2012; $53(3): 839-861$.

30. Harby K, Chiva S, Muñoz-Cobo J. An experimental study on bubble entrainment and flow characteristics of vertical plunging water jets. Experimental Thermal and Fluid Science. $2014 ; 57: 207-220$.

31. Bagatur T. Experimental Analysis of Flow Characteristics from Different Circular Nozzles at Plunging Water Jets. Arabian Journal for Science and Engineering. 2014;39(4):2707-2719.

32. Qu X, Goharzadeh A, Khezzar L, Molki A. Experimental characterization of air-entrainment in a plunging jet. Experimental Thermal and Fluid Science. 2013;44:51-61.

33. Roy AK, Maiti B, Das PK. Visualisation of air entrainment by a plunging jet. Procedia Engineering. 2013;56:468-473.

34. Popinet S. Gerris: a tree-based adaptive solver for the incompressible Euler equations in complex geometries. Journal of Computational Physics. 2003;190(2):572-600.

35. Chorin AJ. Numerical solution of the Navier-Stokes equations. Mathematics of computation. 1968;22(104):745-762.

36. Popinet S. An accurate adaptive solver for surface-tension-driven interfacial flows. Journal of Computational Physics. 2009;228(16):5838-5866.

37. Ervine D, McKeogh E, Elsawy E. Effect of turbulence intensity on the rate of air entrainment by plunging water jets. Proceedings of the Institution of Civil Engineers. 1980;69(2):425-445.

38. Ohkawa A, Kusabiraki D, Kawai Y, Sakai N, Endoh K. Some flow characteristics of a vertical liquid jet system having downcomers. Chemical Engineering Science. 1986;41(9):2347-2361. 